\title{
Dark Shards: velocity substructure from Gaia and direct searches for dark matter
}

\author{
Ciaran A. J. O’Hare, ${ }^{1, *}$ N. Wyn Evans, ${ }^{2,}{ }^{\dagger}$ Christopher McCabe, ${ }^{3,}{ }^{\ddagger}$ GyuChul Myeong,,${ }^{2}$, and Vasily Belokurov ${ }^{2}$ \\ ${ }^{1}$ The University of Sydney, School of Physics, New South Wales, 2006, Australia \\ ${ }^{2}$ Institute of Astronomy, Madingley Rd, Cambridge, CB3 OHA, United Kingdom \\ ${ }^{3}$ Department of Physics, King's College London, Strand, London, WC2R 2LS, United Kingdom
}

(Dated: January 15, 2020)

\begin{abstract}
Data from the Gaia satellite show that the solar neighbourhood of the Milky Way's stellar halo is imprinted with substructure from several accretion events. Evidence of these events is found in "the Shards", stars clustering with high significance in both action space and metallicity. Stars in the Shards share a common origin, likely as ancient satellite galaxies of the Milky Way, so will be embedded in dark matter (DM) counterparts. These "Dark Shards" contain two substantial streams (S1 and S2), as well as several retrograde, prograde and lower energy substructures. The retrograde stream S1 has a very high Earth-frame speed of $\sim 550 \mathrm{~km} \mathrm{~s}^{-1}$ while S2 moves on a prograde, but highly polar orbit and enhances the peak of the speed distribution at around $300 \mathrm{~km} \mathrm{~s}^{-1}$. The presence of the Dark Shards locally leads to modifications of many to the fundamental properties of experimental DM signals. The S2 stream in particular gives rise to an array of effects in searches for axions and in the time dependence of nuclear recoils: shifting the peak day, inducing nonsinusoidal distortions, and increasing the importance of the gravitational focusing of DM by the Sun. Dark Shards additionally bring new features for directional signals, while also enhancing the DM flux towards Cygnus.
\end{abstract}

\section{INTRODUCTION}

The stellar populations of the Milky Way are constructed through two distinct channels: an in situ formation of the bulk of the stars in the disk and bulge of the Galaxy, followed by an ex situ accumulation of substructure that builds most of the stellar halo [1, 2]. The latter stage involves the tidal stripping of stars and dark matter (DM) from surrounding satellites and subhalos and ultimately gives rise to the network of stellar streams that entwine the Milky Way [3-7]. The recent and transformative dataset of astrometry on over a billion stars bestowed upon us by the Gaia satellite [8-10] has brought into focus the extent of this network of streams in the vicinity of Earth [11-15]. This is because Gaia has enabled a vast increase in the number of stars for which six-dimensional phase space coordinates are obtainable. While many of the largest and most famous stellar streams are readily identifiable on the sky from photometry alone [16], phase space information is essential in identifying nearby streams that are dispersed over large areas of the sky.

Here, we use a sample of Gaia main sequence stars in the stellar halo, which are cross-matched with the Sloan Digital Sky Survey (SDSS). The chemo-dynamical structure of the stellar halo has been studied extensively in the last few years. We now know that its velocity ellipsoid exhibits two primary components with striking differences. One is a more metal-poor and essentially isotropic feature associated to the ancient, virialised halo. The other

\footnotetext{
* ciaran.aj.ohare@gmail.com

$\dagger$ nwe@ast.cam.ac.uk

$\ddagger$ christopher.mccabe@kcl.ac.uk

§ gm564@cam.ac.uk
}

is more metal-rich and highly radially anisotropic [1721]. This object was baptised the "Gaia Sausage" after its squashed and elongated shape in velocity space. It is most likely the relic of a major head-on collision between a dwarf spheroidal and the Milky Way approximately 810 billion years ago [19, 22-25]. The aspect of the local stellar halo that has received less detailed study however is the plethora of substructure [11, 12, 26-29]. An extensive list of these substructures, designated "the Shards", was presented recently in Ref. [12], although some of these were anticipated in earlier searches [11, 26, 30, 31].

The substructures are largely at high energy and many are members of the prominent retrograde tail that was known earlier [32]. The list of stellar substructures is surely incomplete and will continue to grow as further searches are made through the Gaia data. Nevertheless, the Shards will likely remain the most prominent examples locally since they have the highest significance and were found in incomplete samples and with brute-force techniques $[11,26]$. Therefore we will use the Shards as typical representatives in the local stellar halo of the ex situ phase of the Milky Way's growth.

Given the observations of the stellar Shards, we expect there to be DM counterparts: the "Dark Shards", relics of the DM subhalos that hosted the progenitors of the Shards. The material in streams remains kinematically cold, so the Dark Shards will have narrow velocity dispersions, smaller than their mean orbital velocities. Hence if even a small fraction of the local DM density is comprised of streams, the resulting speed distribution may be very different from the smooth Maxwellians used by experiments searching for DM on Earth [33-38]. Many methods for detecting DM are highly dependent on, or at the very least require, assumptions for the distribution of particle velocities in the Solar System. High energy, incompletely phase mixed galactic debris accumulated rel- 
atively recently in the Milky Way's growth will make this distribution distinctly non-Gaussian.

The extensive literature (e.g., Refs. [39-61]) on the subject of the astrophysical uncertainties of direct DM detection has typically only investigated the impact of changes to the broad shape of the speed distribution $f(v)$, often inspired by the variety of functional forms observed in simulations [62-65]. Ex situ material has the potential to make more complex modifications to expected signals in DM experiments. While this topic has been discussed somewhat in studies taking inspiration from cosmological simulations [66, 67], this component of the local DM distribution is the most problematic for model-building, given that it encodes all the particularities and peculiarities of the formation of the Milky Way. However, the advent of Gaia and the discovery of substructures like the Shards means that these peculiarities now have an observable counterpart. This data-driven perspective has been followed in several recent studies [68-76]. This work has so far dealt with the shape of $f(v)$ accounting for the isotropic and Sausage components observed in the Gaia stellar halo. Here, our focus is not on the overall shape, but on the fluctuations and deviations from Gaussianity caused by the presence of a host of accreted substructure. While the Dark Shards will be subdominant, their consequences for the velocity distribution are more complex and interesting than simply a shift in functional form.

In terms of stellar content, two of the most substantial Shards are the S1 and S2 streams [12, 26, 77]. Modelling suggests that their progenitors had virial masses $\approx 10^{10} M_{\odot}$ and stellar masses $\approx 10^{6} M_{\odot}$, comparable to modern day dwarf spheroidal satellites like Draco and Ursa Minor. As the S1 stream is counterrotating, its associated DM meets the Earth head-on, so it leaves characteristic signatures in experiments sensitive to high DM speeds [78]. The S2 stellar stream is on a polar orbit that passes through the Earth. Its implications for DM experiments have not hitherto been studied.

To study the effects of the Dark Shards on experimental DM signals in more detail, the earlier sections of this paper are devoted to the refining and remodelling of the Shards from Ref. [12]. We begin in Sec. II by describing our sample of halo stars from the SDSS-Gaia catalogue and then discuss the stellar Shards in detail in Sec. III. We then use the data and our list of substructures to inspire a model for the local DM distribution in Sec. IV. We discuss the new features present in this velocity distribution when observed in the rest frame of the Earth in Sec. V. In the remaining two sections, we detail the consequences of these new features in experiments looking for axions and axion-like particles (Sec. VI), and nuclear recoil-based searches for DM (Sec. VII). In the latter case we also discuss the effects on annual modulation and directional signals. We conclude in Sec. VIII.

A GitHub repository [79] is available which contains links to IPYTHON notebooks for individually reproducing each result.

\section{THE STRUCTURE OF THE LOCAL STELLAR HALO}

The SDSS-Gaia catalogue enhances and recalibrates the astrometric solution of the SDSS's 9th data release [80] with parallaxes and proper motions from Gaia $[8,10]$. This catalogue expands our view of the Milky Way halo out to heliocentric distances of approximately $10 \mathrm{kpc}$. The majority of the radial velocities are sourced from SDSS; however a minor fraction are cross-matched with spectroscopic surveys APOGEE [81], LAMOST DR3 [82] and RAVE-on [83, 84]. Since photometric parallaxes are calculable for main sequence turnoff [85] and blue horizontal branch stars [86], we use these stars to construct a sample of the disk and halo with six-dimensional phase space information out to approximately $10 \mathrm{kpc}$.

To extract the stellar halo from this selection of stars, we adopt a similar set of cuts to those in Refs. [11, 12]. To ensure a high quality sample, stars are selected with photometric parallax distances $<10 \mathrm{kpc}$, a heliocentric radial velocity error $<15 \mathrm{~km} \mathrm{~s}^{-1}$, and distance errors $<2.5 \mathrm{kpc}$. Then, and importantly for this work, stars with clear membership to the galactic thin and thick disks have been removed. Disk stars occupy positive azimuthal velocities, $v_{\phi} \gtrsim 100 \mathrm{~km} \mathrm{~s}^{-1}$ and high metallicities $[\mathrm{Fe} / \mathrm{H}] \gtrsim-1$ (see e.g., Fig. 1 of Ref. [11]). The cut of disk stars is based on these two parameters, but to leave as much of the halo intact as possible it has not been applied too aggressively. Therefore some contamination remains from the metal-poor tail of the thick disk which overlaps strongest with the halo in the $[\mathrm{Fe} / \mathrm{H}]-v_{\phi}$ plane. We will see this contamination for metallicities $[\mathrm{Fe} / \mathrm{H}] \sim-2.1--1.6$. After the cuts, the sample contains 65406 stars.

\section{A. The smooth component}

Figure 1 shows 2-d histograms of the stellar halo sample in $v_{R}$ and $v_{\phi}$, the radial and azimuthal velocities. ${ }^{1}$ We divide the total sample into nine histograms, with cuts on the height above or below the disk $|z|$ and iron abundance $[\mathrm{Fe} / \mathrm{H}]$, as indicated by the black dashed lines above and to the right of the nine histograms. A striking observation can be made when the metal rich panels (right) are compared with the metal-poor panels (left): the distribution of stars is clearly extended in $v_{R}$ in the right-most panels, making it sausage-shaped. In contrast, the distribution of stars in the left-most panels is circular, consistent with a roundish, isotropic halo. We will refer to this as the "Round Halo" to distinguish it from the radially anisotropic "Sausage". Some contami-

\footnotetext{
1 Throughout, we use $(R, \phi, z)$ as galactocentric cylindrical polar coordinates and $(r, \theta, \phi)$ as galactocentric spherical polars.
} 


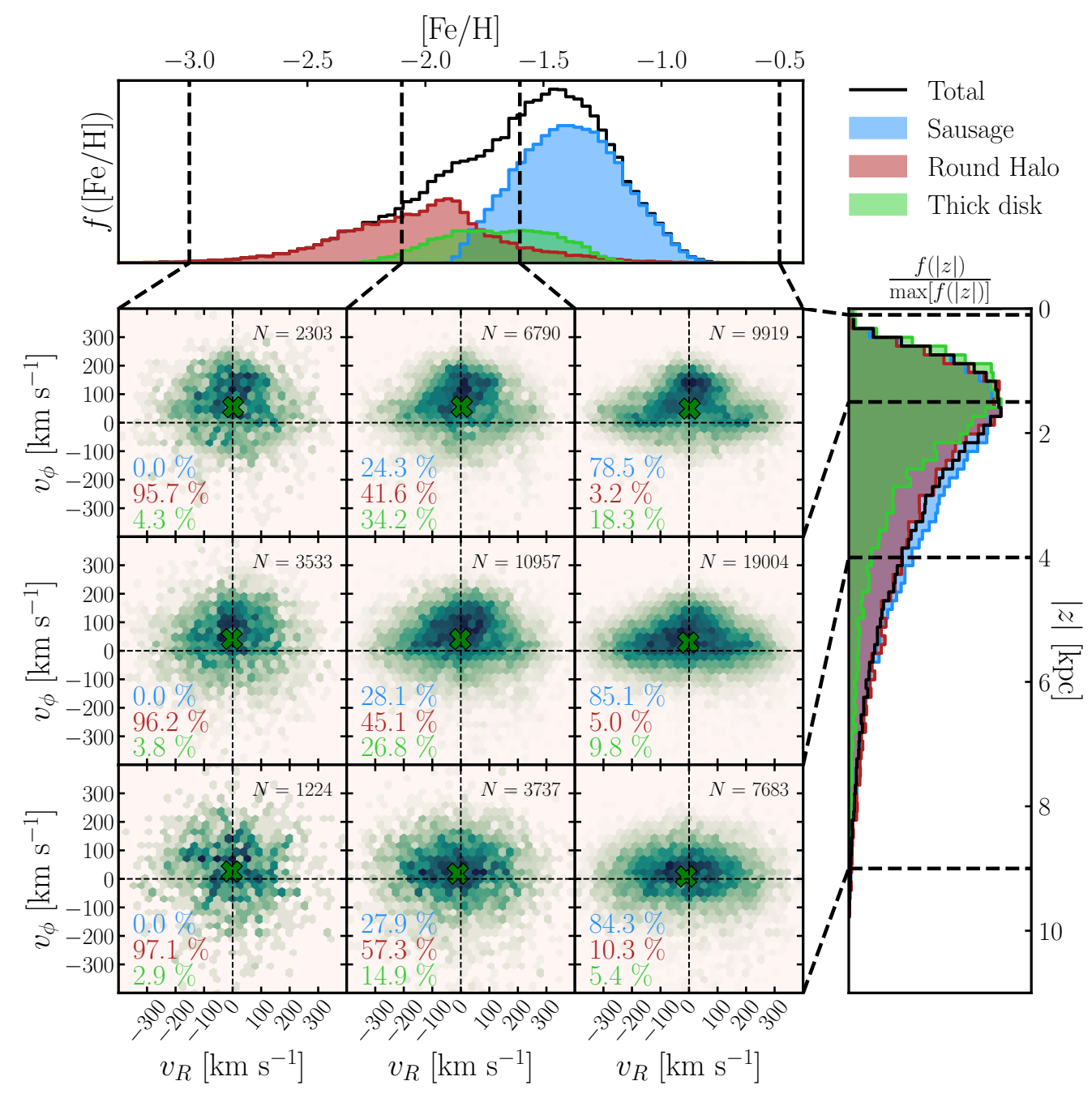

FIG. 1. Kinematic structure of the stellar halo sample. The central panels show 2-d histograms of the radial and azimuthal velocities, $v_{R}$ and $v_{\phi}$, of the SDSS-Gaia stellar halo sample used in this paper. Darker colours represent a higher star count. The full sample has been divided into nine bins in terms of metallicity $([\mathrm{Fe} / \mathrm{H}])$ and distance away from the disk plane $(|z|)$, as indicated by the dashed black lines (following Ref [19]). Reading from left to right corresponds to increasing [Fe/H], while from top to bottom corresponds to increasing $|z|$. The green cross shows the mean velocity in each bin, while $N$ gives the number of stars (there are 261 stars with $[\mathrm{Fe} / \mathrm{H}]<-3$ or $|z|>9$ that are not included in the 2-d histograms). We decompose the sample into three components: Round Halo, Sausage and Thick disk. Their 1-d $[\mathrm{Fe} / \mathrm{H}]$ and $|z|$ distributions are shown above and to the right of the histograms. The fraction of each component in the nine bins is given by the coloured percentage values.

nation by thick disk stars can also be seen in the top-row middle panel at $v_{\phi} \simeq 125 \mathrm{~km} \mathrm{~s}^{-1}$ and $v_{R} \simeq 0.0 \mathrm{~km} \mathrm{~s}^{-1}$.

Above and to the right of the 2-d histograms, we show the 1-d histograms of $|z|$ and $[\mathrm{Fe} / \mathrm{H}]$ for the three components: the Round Halo (red), Sausage (blue) and thick disk (green). To partition the stars in this way, we have decomposed the sample by applying a Gaussian mixture model using the stellar velocity and metallicity data: $\mathbf{q}=\left\{v_{R}, v_{\phi}, v_{z},[\mathrm{Fe} / \mathrm{H}]\right\}$. The probability density function for the three components that we fit to the stellar sample is,

$$
\begin{aligned}
f(\mathbf{q})=\sum_{i=1}^{3} & \frac{w_{i}}{\sqrt{(2 \pi)^{D} \operatorname{det} \boldsymbol{\Sigma}_{i}}} \\
& \times \exp \left(-\frac{1}{2}\left(\mathbf{q}-\overline{\mathbf{q}}_{i}\right)^{T} \boldsymbol{\Sigma}_{i}^{-1}\left(\mathbf{q}-\overline{\mathbf{q}}_{i}\right)\right)
\end{aligned}
$$

where $\boldsymbol{\Sigma}_{i}$ is a covariance matrix for component $i, w_{i}$ is its relative weight, and the exponent $D$ is the dimensionality of the data, which in this case is four. In Table I, we give the means, weights and covariances for this decomposition for the complete stellar halo sample.

As has been noted before [18-20, 87], the Sausage component dominates the local stellar halo in main sequence 


\begin{tabular}{l|lll}
\hline \hline & Round Halo & Sausage & Thick disk \\
\hline $\bar{v}_{r}\left[\mathrm{~km} \mathrm{~s}^{-1}\right]$ & -0.1 & -8.2 & 12.4 \\
$\bar{v}_{\phi}\left[\mathrm{km} \mathrm{s}^{-1}\right]$ & 6.0 & 25.7 & 128.8 \\
$\bar{v}_{z}\left[\mathrm{~km} \mathrm{~s}^{-1}\right]$ & 8.0 & 0.99 & 3.6 \\
$\sigma_{r}\left[\mathrm{~km} \mathrm{~s}^{-1}\right]$ & 144.4 & 158.9 & 76.8 \\
$\sigma_{\phi}\left[\mathrm{km} \mathrm{s}^{-1}\right]$ & 120.0 & 61.5 & 50.1 \\
$\sigma_{z}\left[\mathrm{~km} \mathrm{~s}^{-1}\right]$ & 115.7 & 80.9 & 62.7 \\
$\Sigma_{r \phi} / \sigma_{r} \sigma_{\phi}$ & 0 & 0 & 0 \\
$\Sigma_{r z} / \sigma_{r} \sigma_{z}$ & 0.1 & 0.3 & 0.2 \\
$\Sigma_{\phi z} / \sigma_{\phi} \sigma_{z}$ & 0 & 0 & 0 \\
$\overline{[\mathrm{Fe} / \mathrm{H}]}$ & -1.9 & -1.4 & -1.7 \\
$\sigma_{[\mathrm{Fe} / \mathrm{H}]}$ & 0.4 & 0.2 & 0.3 \\
$w$ & 0.33 & 0.51 & 0.16 \\
\hline \hline
\end{tabular}

TABLE I. Results of a three population Gaussian mixture model fit to the SDSS-Gaia stellar halo sample. In order, we show the mean velocities, velocity dispersions (diagonal covariance matrix elements), the off-diagonal covariance matrix elements for the velocities, mean metallicity, standard deviation on the metallicity, and finally the weighting of each component $w$. The thick disk is included as a third population to model its small contamination of the sample.

stars. It has also been identified independently with other tracers including blue horizontal branch stars [86, 88], RR Lyraes [22, 89] and K giants [90]. The characteristic range of metallicities, $[\mathrm{Fe} / \mathrm{H}] \approx-1.4$, means that its stars are slightly more metal-rich than the halo average $(\approx-1.9)$ but metal-poor relative to the (thin and thick) disks $(\approx-0.8)$. This strongly suggests that the Sausage component is the remnant of a merger in the Milky Way's recent past. The unusual kinematic profile implies that the merger was a head-on collision with an object on a low inclination orbit [19]. The progenitor Sausage galaxy most likely had a total mass $\gtrsim 10^{10} M_{\odot}$ and was accreted around redshift $\lesssim 3[19,24,91]$.

\section{B. The Shards}

The Round Halo and the Sausage are apparent when visualising the full sample in velocity space. The importance of the ex-situ halo, the accreted part of our Galaxy, on the other hand is more evident in action space [92]. Actions are adiabatic invariants so are conserved under slow evolution of the potential. This means that stars belonging to a discrete bound object that are accreted together slowly will remain clustered in action space even if they are too dispersed or too close to us to be identified otherwise. Searching through action space is therefore the most efficient and powerful way to find local substructure. In addition to the three actions $\left(J_{R}, J_{\phi}, J_{z}\right)$, another useful quantity is the orbital energy, $E$, as substructure from recent in-falls is usually at high energy. The distribution of the full sample in these three ac- tions versus energy are shown in the top row of Fig. 2. Actions are computed using the numerical methods reviewed in Ref. [93] and the updated Milky Way potential from McMillan [94].

The action space distribution of the sample contains abundant substructure which is highlighted in the top row of Fig. 2. The 30 highest significance clusters were initially listed in Ref. [12]; however the list in fact extends to a total of 69 . We call all these action-space clusters "Shards" throughout our paper. Action space clusterings are typically associated with kinematically cold streams, however only a fraction of the reported Shards are significant enough for this interpretation to be ascribed. Those action space clusters that are not focused tightly around single streaming velocities are sometimes referred to as "clumps", "objects" or "moving groups" [11]. For example our list of Shards include the prominent S1 and S2 streams as well as the clump $\mathrm{C} 2$, themselves rediscoveries of earlier reported objects found in phase space [11, 26]. For clarity, we refer to S1 and S2 as "streams", and simply use the term "substructures" for the remaining Shards. In Fig. 2 we grouped them together into two categories "Retrograde" and "Prograde" according to the sign of their $J_{\phi}$ (these correspond to the $\mathrm{Rg}$ and Cand labels used in Ref. [11]). In total, the Shards comprise a subset of 1117 stars in the Gaia-SDSS halo sample. ${ }^{2}$

The Retrograde Shards contain some of the highest energy stars in the halo and seem to be comparatively metal-rich, with $-1.9 \lesssim[\mathrm{Fe} / \mathrm{H}] \lesssim-1.3$. While a similar range of metallicities is found for the Sausage stars, the characteristic abundances of other $\alpha$-elements in the retrograde tail clearly distinguishes them in origin from the Sausage galaxy [95-97]. It has been suggested that this retrograde tail and the substructures it contains may all be associated with another major lowinclination accretion of a $10^{10} M_{\odot}$ dwarf galaxy, dubbed the "Sequoia" [98]. This interpretation is bolstered by several globular clusters on retrograde orbits that we have have also marked on Fig. 2. These include the extended globular cluster FSR1758 [99, 100] —from which the arboreal moniker originates - as well as the anomalous $\omega$ Centauri, which has for many years been believed to have begun life as the nucleus of a dwarf galaxy [101]. These clusters may have all originally been part of the Sequoia galaxy. One of the most important Shards, S1 - already the subject of some discussion in the literature [78, 102, 103] — appears to be associated with the globular cluster NGC 3201 which may have resided within the Sequoia.

\footnotetext{
${ }^{2}$ While the Shards were originally identified in the Gaia-SDSS sample with Gaia-DR1 proper motions, we have verified that the fitted parameters of the S1 and S2 streams are essentially unchanged when the slightly improved Gaia-DR2 is used. This is likely to be the case for the majority of the Shards.
} 

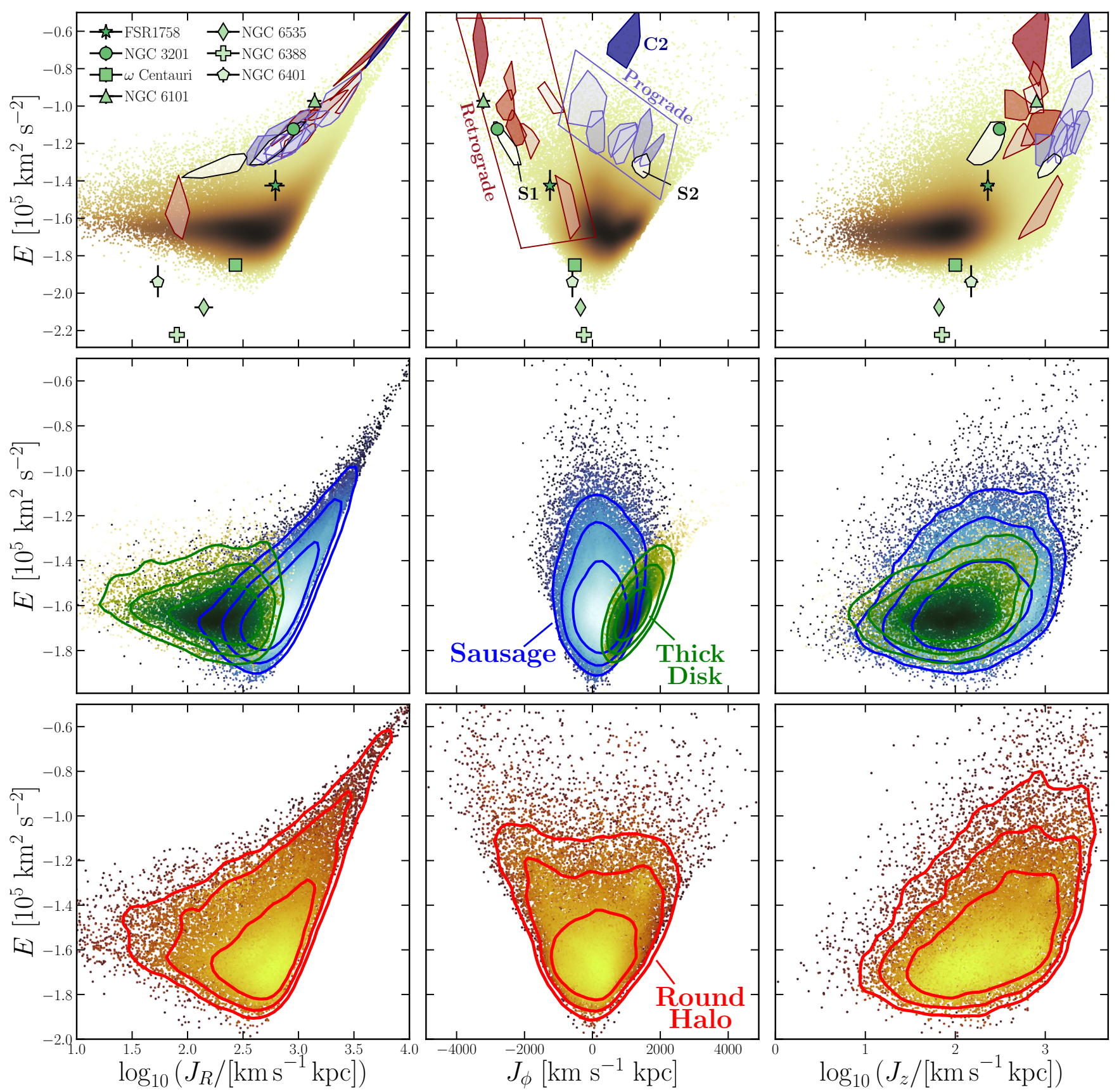

FIG. 2. Action-energy distributions for the full sample of stars (top row) and after being partitioned into the Sausage and Thick Disk (middle row), and Round Halo (bottom row). In the top row, we show the convex hulls of stars belonging to the Shards. We highlight the three highest significance Shards (S1, S2 and C2) and group the retrograde and prograde candidate substructures together. The middle and bottom rows show subsets of the full sample that are partitioned according to the decomposition used in Fig. 1 and summarised in Table I. We show logarithmically spaced contours over the distributions to highlight the general shapes, for example the high energy retrograde tail of negative $J_{\phi}$ in the bottom row. In the top row we also show the seven retrograde globular clusters that are potentially linked with the Sequoia event producing S1 and the retrograde tail.

\section{MODELLING THE SHARDS}

We fit each Shard with a Gaussian mixture model based on available data: $\mathbf{q}=\left\{X, Y, Z, v_{R}, v_{\phi}, v_{z},[\mathrm{Fe} / \mathrm{H}]\right\}$, i.e., $D=7$ in Eq.(1). We include spatial information here to improve the measurement of the trajectory of the stream and to allow us to estimate the velocity at the solar position. While a Gaussian is a good description of a stream's cross section, it is not usually used to describe 

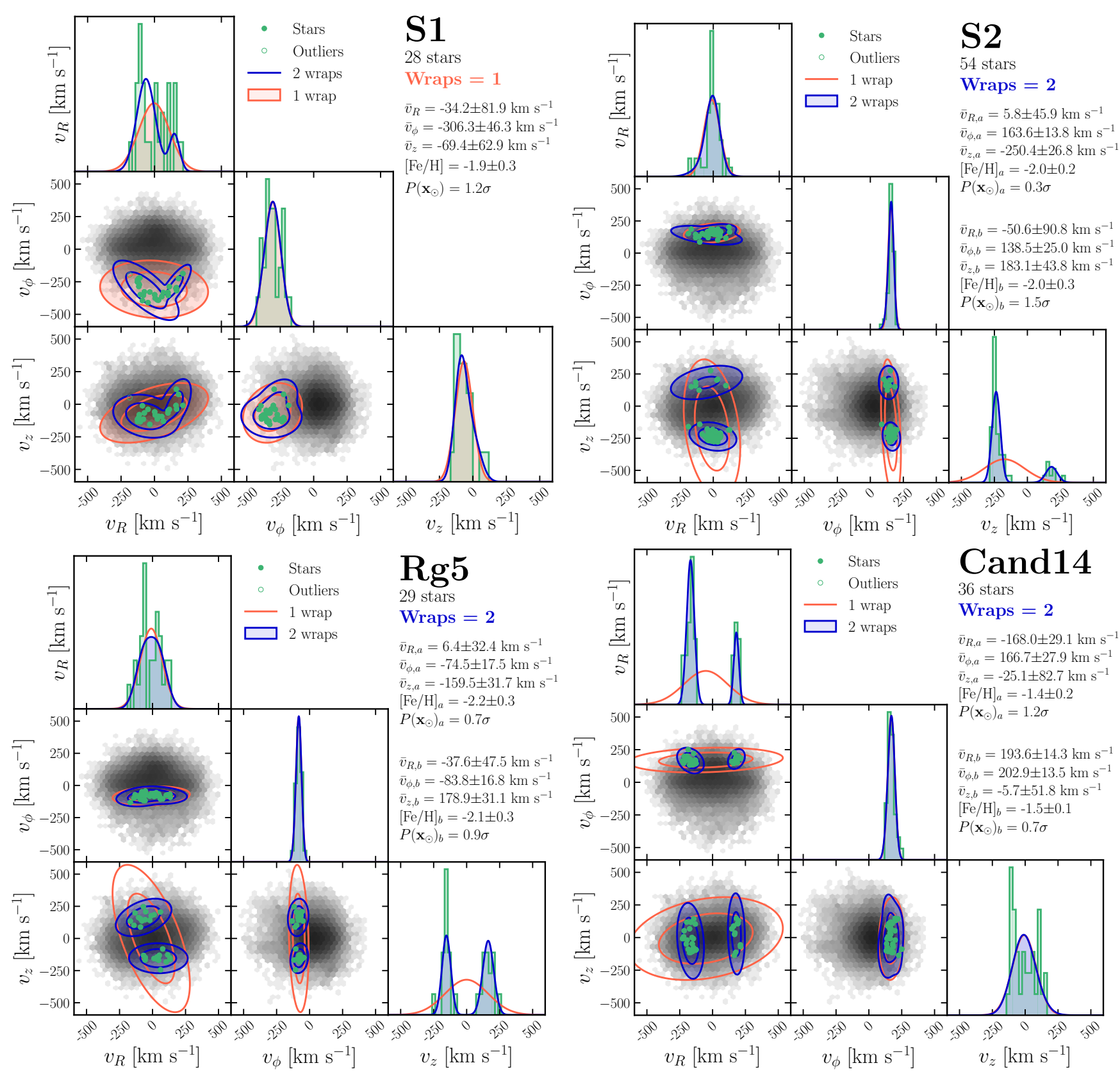

FIG. 3. Distributions of stellar velocities in galactic cylindrical coordinates for four Shards: S1, S2, Rg5 and Cand14. The velocity of stars associated with each Shard are shown as green points. Fits to the seven-dimensional (position, velocity and metallicity) data with the Gaussian mixture model [Eq.(1)] are shown: the orange contour indicates an $n=1$ Gaussian whereas the blue contours show the $n=2$ case. The extent of the SDSS-Gaia sample is shown in grey. For each Shard, we select the number of wraps by using the minimum Bayesian information criterion. S1 is better fit by one wrap, while S2, Rg5 and Cand14 are better fit by two wraps. The best-fit parameters are given in the accompanying text. The significance value $P\left(\mathbf{x}_{\odot}\right)$ quantifies how far away the solar position is relative to the spatial distribution of each Shard.

the shape along the stream. However, given the small extent of the SDSS-Gaia footprint relative to the size of the apocentric radii of the stellar orbits, this model should be fine. Ultimately the process of including the stellar spatial positions is only to be able to remove those Shards which clearly do not intersect the solar position.

Many clusters of stars in action space correspond to separated clusters in velocity space coordinates. So we vary the number of possible populations, $n$ in Eq.(1). In Fig. 3, we show the stellar velocity data for four Shards fitted to these models. We choose the two highest significance streams (S1 and S2) and two examples of retrograde and prograde substructures (Rg5 and Cand14 respectively). 

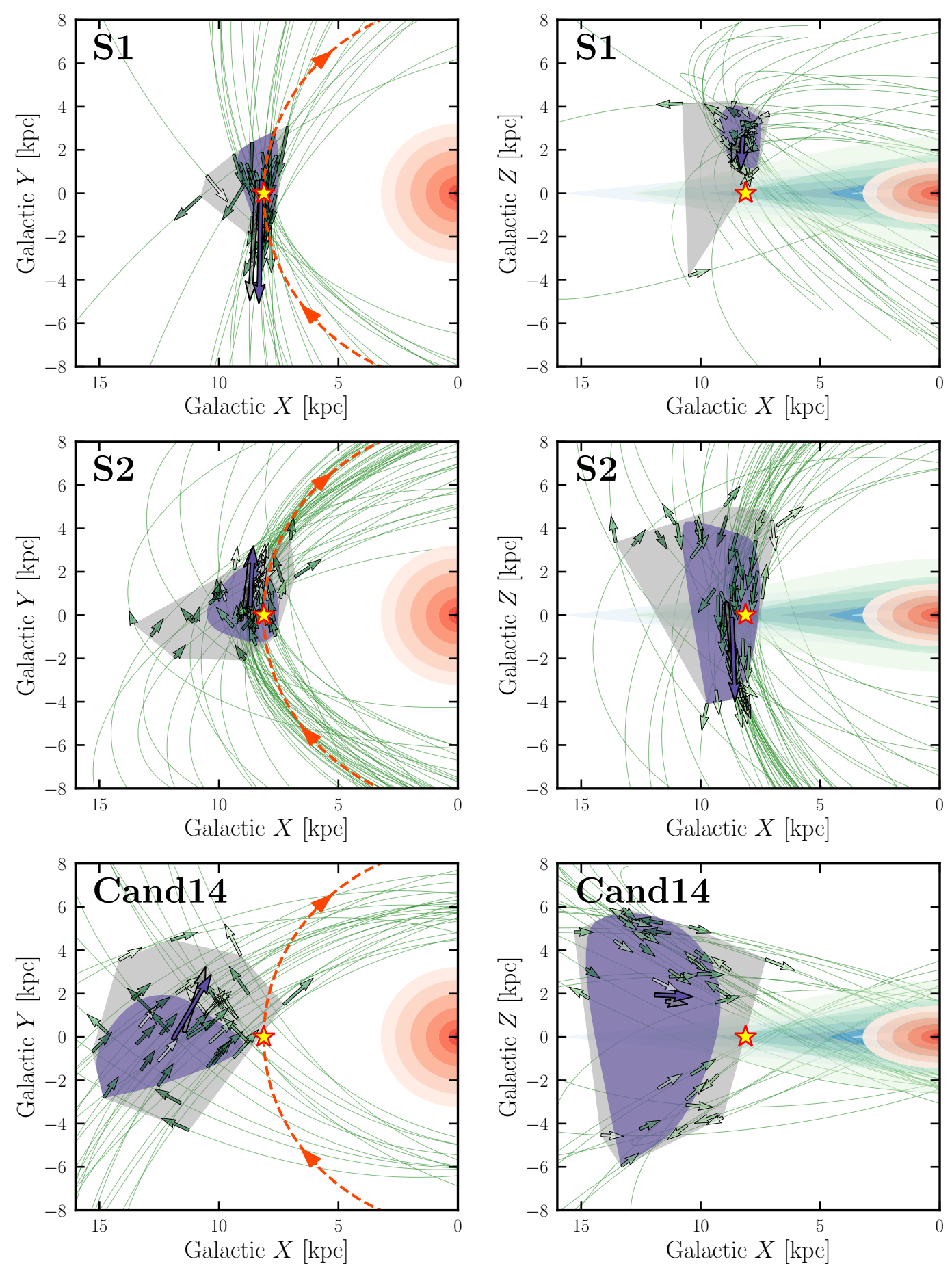

FIG. 4. Stellar positions, velocities and orbits in the galactic $X-Y$ and $X-Z$ planes for three Shards: S1, S2 and Cand14 (from top to bottom). The orange region traces the shape of the density profile of the galactic bulge whereas the greenish and blueish regions correspond to the thin and thick disk respectively. The convex hull for all the stellar positions is shown as the outer gray shaded region, within which we show a reduced region after performing a $z$-score cut of spatial outliers. The orbit and position of the Sun is shown as an orange-red dashed line and yellow star. The orbits of the each star integrated forward and backward in time by 100 Myr are shown as green lines. 


\begin{tabular}{|c|c|c|c|c|c|c|c|c|}
\hline \multicolumn{2}{|r|}{ Name } & $\begin{array}{l}\text { Number } \\
\text { of stars }\end{array}$ & $\begin{array}{c}(X, Y, Z) \\
\mathrm{kpc}\end{array}$ & $\begin{array}{l}(\Delta X, \Delta Y, \Delta Z) \\
\mathrm{kpc}\end{array}$ & $\begin{array}{c}\left(v_{R}, v_{\phi}, v_{z}\right) \\
\mathrm{km} \mathrm{s}^{-1}\end{array}$ & $\begin{array}{c}\left(\sigma_{R}, \sigma_{\phi}, \sigma_{z}\right) \\
\mathrm{km} \mathrm{s}^{-1}\end{array}$ & $\langle[\mathrm{Fe} / \mathrm{H}]\rangle$ & $\begin{array}{l}P\left(\mathbf{x}_{\odot}\right) \\
(\sigma)\end{array}$ \\
\hline $\bar{n}$ & & 28 & $(8.4,0.6,2.6)$ & $(0.7,1.8,2.2)$ & $(-34.2,-306.3,-64.4)$ & $\overline{(81.9,46.3,62.9)}$ & $-1.9 \pm 0.3$ & 1.2 \\
\hline \multirow{2}{*}{ N } & $\bar{a}$ & 46 & $\begin{array}{l}(8.7,0.4,0.1) \\
\end{array}$ & 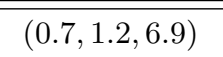 & $(5.8,163.6,-250.4)$ & (45.9,13.8,26.8) & $-2.0 \pm 0.2$ & 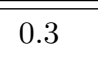 \\
\hline & $\mathrm{b}$ & 8 & $(10.1,0.2,3.3)$ & $(4.9,0.7,1.4)$ & $(-50.6,138.5,183.1)$ & $(90.8,25.0,43.8)$ & $-2.0 \pm 0.3$ & 1.5 \\
\hline \multirow{7}{*}{ 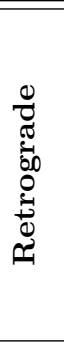 } & Rg2 & 13 & $(8.9,0.3,4.4)$ & $(0.8,2.1,2.7)$ & $(44.5,-248.4,185.2)$ & $(105.9,23.1,63.5)$ & $-1.6 \pm 0.2$ & 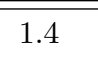 \\
\hline & Rg5a & 15 & $(8.4,0.8,1.1)$ & $(1.0,1.3,3.3)$ & $(6.4,-74.5,-159.5)$ & $(32.4,17.5,31.7)$ & $-2.2 \pm 0.3$ & 0.7 \\
\hline & Rg5b & 14 & $(8.1,-0.2,2.2)$ & $(1.1,1.2,2.4)$ & $(-37.6,-83.8,178.1)$ & $(47.5,16.8,31.1)$ & $-2.1 \pm 0.3$ & 0.9 \\
\hline & Rg6a & 17 & $(8.3,0.2,3.3)$ & $(1.8,1.4,2.0)$ & $(105.1,-230.2,202.4)$ & $(73.7,16.8,86.6)$ & $-1.6 \pm 0.2$ & 1.1 \\
\hline & Rg6b & 12 & $(8.5,0.9,3.2)$ & $(1.5,1.5,2.2)$ & $(-233.2,-221.8,51.6)$ & $(32.7,14.4,115.7)$ & $-1.7 \pm 0.3$ & 0.6 \\
\hline & Rg7a & 5 & $(8.2,0.5,3.3)$ & $(2.1,1.5,3.3)$ & $(309.0,-191.3,-83.4)$ & $(66.7,17.1,102.7)$ & $-1.5 \pm 0.1$ & 1.1 \\
\hline & Rg7b & 9 & $(8.9,-0.0,5.1)$ & $(1.9,1.3,2.0)$ & $(-288.7,-158.1,-105.5)$ & $(78.7,65.8,111.8)$ & $-1.5 \pm 0.3$ & 1.8 \\
\hline \multirow{15}{*}{ 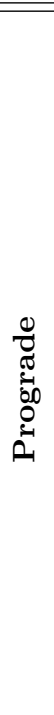 } & Cand8a & 31 & $(9.9,-0.1,2.4)$ & 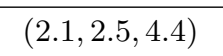 & $(-6.7,207.7,-186.4)$ & $\begin{array}{l}(114.6,20.8,73.5) \\
\end{array}$ & $-1.8 \pm 0.4$ & 0.4 \\
\hline & Cand8b & 18 & $(8.4,0.6,1.1)$ & $(1.5,2.2,3.6)$ & $(33.6,213.9,214.1)$ & $(96.5,22.7,37.7)$ & $-1.8 \pm 0.2$ & 0.1 \\
\hline & Cand9 & 43 & $(9.2,-0.2,1.7)$ & $(1.1,1.4,3.4)$ & $(11.0,177.5,-251.4)$ & $(120.6,13.9,132.2)$ & $-1.8 \pm 0.2$ & 0.5 \\
\hline & Cand10 & 38 & $(8.6,-0.0,2.0)$ & $(1.7,1.3,2.5)$ & $(-37.4,20.0,192.3)$ & $(161.5,18.2,195.0)$ & $-2.0 \pm 0.2$ & 0.2 \\
\hline & Cand11a & 14 & $(9.1,-0.3,2.7)$ & $(2.5,1.4,3.8)$ & $(36.8,116.5,-271.5)$ & $(96.1,27.9,95.4)$ & $-2.1 \pm 0.3$ & 0.3 \\
\hline & Cand11b & 23 & $(9.0,-0.1,2.4)$ & $(1.9,1.1,2.8)$ & $(-152.7,80.2,258.2)$ & $(122.1,21.0,38.9)$ & $-2.0 \pm 0.3$ & 0.5 \\
\hline & Cand12 & 36 & $(9.6,-0.8,3.7)$ & $(2.0,2.4,4.2)$ & $(-43.3,102.4,50.0)$ & $(172.8,21.2,197.8)$ & $-1.6 \pm 0.2$ & 0.6 \\
\hline & Cand13 & 36 & $(9.1,1.0,3.1)$ & $(2.5,2.0,4.1)$ & $(-2.1,-13.2,202.2)$ & $(215.7,28.1,215.9)$ & $-1.4 \pm 0.2$ & 0.4 \\
\hline & Cand14a & 24 & $(11.9,0.2,1.8)$ & $(1.8,1.7,3.6)$ & $(-168.0,166.7,-25.1)$ & $(29.1,27.9,82.7)$ & $-1.4 \pm 0.2$ & 1.2 \\
\hline & Cand14b & 12 & $(10.7,0.3,1.4)$ & $(1.8,2.1,3.5)$ & $(193.6,202.9,-5.7)$ & $(14.3,13.5,51.8)$ & $-1.5 \pm 0.1$ & 0.7 \\
\hline & Cand15a & 12 & $(10.5,1.4,4.0)$ & $(1.9,2.1,3.9)$ & $(-297.4,220.0,-49.9)$ & $(29.6,23.5,79.3)$ & $-1.5 \pm 0.1$ & 1.2 \\
\hline & Cand15b & 7 & $(10.3,-0.3,2.4)$ & $(1.8,2.3,5.9)$ & $(291.3,207.3,48.3)$ & $(20.2,10.4,68.7)$ & $-1.4 \pm 0.1$ & 0.5 \\
\hline & Cand16a & 12 & $(8.7,0.5,3.9)$ & $(1.6,1.5,3.9)$ & $(315.2,109.2,-12.5)$ & $(30.9,4.6,67.2)$ & $-1.4 \pm 0.2$ & 0.7 \\
\hline & Cand16b & 5 & $(8.9,2.8,-1.3)$ & $(1.3,2.1,3.2)$ & $(-360.7,147.5,81.7)$ & $(26.7,9.2,76.3)$ & $-1.4 \pm 0.1$ & 0.9 \\
\hline & Cand17 & 10 & $(9.5,-0.4,2.0)$ & $(1.0,0.9,2.5)$ & $(127.6,68.0,339.4)$ & $(157.4,8.0,54.8)$ & $-2.1 \pm 0.2$ & 0.7 \\
\hline
\end{tabular}

TABLE II. Basic parameters of the Shards. Those containing two populations in phase space are divided into two items "a" and "b". The Shards are organised into five categories: S1, S2, Retrograde, Prograde and Low-Energy (the latter group listed in Table III for brevity). The significance of the location of the Sun is given in $\sigma$ in the final column: a smaller number implies that the substructure spatial distribution overlaps the Sun.

To quantify how distant each Shard is from Earth, we also display $P\left(\mathbf{x}_{\odot}\right)$, the significance of the solar position relative to the spatial distribution of the stars. We take the solar position in galactocentric rectangular coordinates to be $\mathbf{x}_{\odot}=(8.2,0,0.014) \mathrm{kpc}[94,104,105]$ and calculate the significance of this position in the Gaussian fit to the distribution of stellar locations.

To evaluate the most appropriate number of populations, we select the option which yields the minimum Bayesian information criterion, although in most cases this choice is visible by eye. For three of the four examples shown, we find that there are two clear subpopulations. These represent the leading and trailing tails of stellar streams, which can stretch over large distances and wrap the Galaxy multiple times. The power of action space searches for substructure is that they allow a single object to be identified, whereas a search in velocity space would have typically found two distinct clusters. The
Shards that do not exhibit this bimodal feature instead are observable because they are close to the pericentric passage of the stream. In these cases, we are positioned close to the maximum stellar density along the stream. Figure 4 visualises this idea. We plot the individual stellar positions and velocities for three Shards and integrate the orbits of those stars forward and backward in time by 100 Myr using MWPotential2014 in the galpy package [106].

In the first two examples, we see that stars are on orbits that are grouped together tightly and are at a position very close to pericentre. In the bottom row of Fig. 4, however, Cand14 has at least two wraps coinciding with the solar position. The stars in this case are located at positions much larger than the mean pericentre of their orbits. This illustrates the interpretation discussed above, that the Shards consist of (i) streams with pericentres close to the solar position, and (ii) streams with multiple 
wrappings coinciding with the solar position.

A complete summary of the fit to each of the main substructures is given in Table II. There is another, longer list of the lower-significance (and typically lower-energy) Shards which we have included in Appendix A. There are two streams in the Shards, labelled S1 and S2. The remaining substructures tagged as "Rg" and "Cand" correspond to the categories Retrograde and Prograde. We now extend the naming convention of Ref. [12] to indicate those streams with multiple wraps entering the survey footprint. We add the suffix "a" and "b" in those cases. This subtlety is a key difference between this list and that of Ref. [12]. Accounting for multiple wraps prevents us overestimating the velocity dispersions of the substructures which could be anomalously large otherwise.

In Table II, we also listed $P\left(\mathbf{x}_{\odot}\right)$, the significance of the solar position relative to the spatial distribution of the stars. In the next Section, we will devise a model for the Dark Shards in the velocity distribution. For this, we select only those candidates with significances $<2$ and populations $>4$. This means that only the most important Shards for DM searches are considered. Notably $\mathrm{C} 2$, which has a relatively high significance in action space, is located too far away from the Sun to be included in our model. The Shards that remain are those listed in Tables II and III, a total of 59 .

To streamline our discussion even further, we organise the Shards into five categories: S1, S2, Retrograde, Prograde and Low Energy. S1 and S2 are given their own status to reflect their very high significance as clusters in action space. The Retrograde Shards all have similar significances that are lower than S1 and S2, but since they share kinematic and chemical properties it is reasonable to group them together. This is also the case for the Prograde category. The Low Energy category consists of all of the remaining lower significance Shards listed in Table III.

\section{DARK SHARDS IN THE HALO MODEL}

The DM associated with the Shards - the Dark Shards - will change the local velocity distribution of DM and therefore impact the observable signals in direct detection experiments on Earth. In this section, utilising the properties of the Shards in Table II, we construct a simple model for the Milky Way DM halo to study the effects of the Dark Shards.

Our model consists of three components: the roundish, isotropic halo $f_{\mathrm{R}}(\mathbf{v})$; the Gaia Sausage $f_{\mathrm{S}}(\mathbf{v})$; and the Dark Shards $f_{\xi}(\mathbf{v})$. The velocity distribution in the galactic frame is the sum of the three components,

$$
f_{\text {gal }}(\mathbf{v})=\xi_{\mathrm{R}} f_{\mathrm{R}}(\mathbf{v})+\xi_{\mathrm{S}} f_{\mathrm{S}}(\mathbf{v})+\sum_{i=1}^{n_{\xi}} \xi_{i} f_{\xi}^{i}(\mathbf{v})
$$

where the sum extends over the $n_{\xi}$ Dark Shards and $\xi_{\mathrm{R}}, \xi_{\mathrm{S}}$ and $\xi_{i}$ give the fractional weighting to the local
DM density in the Round Halo, Sausage and each Shard, respectively. By definition, $\xi_{\mathrm{R}}+\xi_{\mathrm{S}}+\xi_{\text {tot }}=1$, where $\xi_{\text {tot }}=\sum_{i=1}^{n_{\xi}} \xi_{i}$. We will first discuss the functional forms for $f_{\mathrm{R}}(\mathbf{v}), f_{\mathrm{S}}(\mathbf{v})$ and $f_{\xi}^{i}(\mathbf{v})$ before turning to discuss the weighting that we assign to each component.

\section{The Round DM Halo}

We model the velocity distribution of the Round DM Halo as a smooth, isotropic Gaussian distribution [107]:

$$
\begin{aligned}
& f_{\mathrm{R}}(\mathbf{v})=\frac{1}{\left(2 \pi \sigma_{v}^{2}\right)^{3 / 2} N_{\mathrm{R}, \mathrm{esc}}} \exp \left(-\frac{|\mathbf{v}|^{2}}{2 \sigma_{v}^{2}}\right) \\
& \times \Theta\left(v_{\mathrm{esc}}-|\mathbf{v}|\right) .
\end{aligned}
$$

Here, $\sigma_{v}$ is the velocity dispersion which is set, at the solar position, by the value of the local standard of rest $v_{0}=\sqrt{2} \sigma_{v}=235 \mathrm{~km} \mathrm{~s}^{-1}$. We have adjusted the value of $v_{0}$ from Ref. [72] by taking into account the more recent determination of the distance to the galactic centre, $R_{0}$ [105]. We truncate the velocity distribution at the galactic escape speed $v_{\text {esc }}$, using the Heaviside function $\Theta$. The constant $N_{\mathrm{R} \text {,esc }}$ ensures the velocity distribution remains normalised to unity

$$
N_{\mathrm{R}, \mathrm{esc}}=\operatorname{erf}\left(\frac{v_{\mathrm{esc}}}{\sqrt{2} \sigma_{v}}\right)-\sqrt{\frac{2}{\pi}} \frac{v_{\mathrm{esc}}}{\sigma_{v}} \exp \left(-\frac{v_{\mathrm{esc}}^{2}}{2 \sigma_{v}^{2}}\right) .
$$

A recent analysis that used a local sample of approximately 2300 high velocity counterrotating stars and a prior distribution inspired by numerical simulations obtained the value $v_{\text {esc }}=528_{-25}^{+24} \mathrm{~km} \mathrm{~s}^{-1}$ [108], in agreement with, but improving upon, earlier determinations $[109,110]$.

\section{The Sausage}

We follow the $\mathrm{SHM}^{++}[72]$ and model the Sausage component with a triaxial Gaussian velocity distribution,

$$
\begin{aligned}
f_{\mathrm{S}}(\mathbf{v})=\frac{1}{(2 \pi)^{3 / 2} \sigma_{r} \sigma_{\theta}^{2} N_{\mathrm{S}, \mathrm{esc}}} \exp \left(-\frac{v_{r}^{2}}{2 \sigma_{r}^{2}}\right. & \left.-\frac{v_{\theta}^{2}}{2 \sigma_{\theta}^{2}}-\frac{v_{\phi}^{2}}{2 \sigma_{\phi}^{2}}\right) \\
& \times \Theta\left(v_{\mathrm{esc}}-|\mathbf{v}|\right) .
\end{aligned}
$$

The velocity dispersions are related to the local standard of rest, $v_{0}=235 \mathrm{~km} \mathrm{~s}^{-1}$, by [111]

$$
\sigma_{r}^{2}=\frac{3 v_{0}^{2}}{2(3-2 \beta)}, \quad \sigma_{\theta}^{2}=\sigma_{\phi}^{2}=\frac{3 v_{0}^{2}(1-\beta)}{2(3-2 \beta)},
$$

where $\beta$ is the anisotropy parameter. We assume that $\beta$ for the DM in the Sausage is the same as the value for the stars, $\beta=0.9[18,19]$. The escape speed normalisation 
modifier for this distribution is [72],

$$
\begin{aligned}
N_{\mathrm{S}, \mathrm{esc}}=\operatorname{erf}\left(\frac{v_{\mathrm{esc}}}{\sqrt{2} \sigma_{r}}\right) & -\sqrt{\frac{1-\beta}{\beta}} \exp \left(-\frac{v_{\mathrm{esc}}^{2}}{2 \sigma_{\theta}^{2}}\right) \\
& \times \operatorname{erfi}\left(\frac{v_{\mathrm{esc}}}{\sqrt{2} \sigma_{r}} \cdot \sqrt{\frac{\beta}{1-\beta}}\right) .
\end{aligned}
$$

Since the $\mathrm{SHM}^{++}$is a model that contains only $f_{\mathrm{R}}$ and $f_{\mathrm{S}}$, we refer to the case when $\xi_{\text {tot }}=0$ as the $\mathrm{SHM}^{++}$.

\section{The Dark Shards}

In the context of this paper, $f_{\xi}^{i}(\mathbf{v})$ is the most important component in Eq.(2) as it models the Dark Shards. We write each subcomponent as

$$
\begin{aligned}
f_{\xi}^{i}(\mathbf{v})=\frac{1}{\left(8 \pi^{3} \operatorname{det} \boldsymbol{\sigma}_{\xi}^{i 2}\right)^{1 / 2} N_{\xi}, \mathrm{esc}} \\
\quad \times \exp \left(-\left(\mathbf{v}-\mathbf{v}_{\xi}^{i}\right)^{T} \frac{\left(\boldsymbol{\sigma}_{\xi}^{i}\right)^{-2}}{2}\left(\mathbf{v}-\mathbf{v}_{\xi}^{i}\right)\right) \\
\quad \times \Theta\left(v_{\mathrm{esc}}-|\mathbf{v}|\right) .
\end{aligned}
$$

Compared with the Round Halo and the Gaia Sausage, the Dark Shards do not have zero mean velocity in the galactic frame of reference. Instead, the triaxial velocity distribution is offset by the velocity of the moving substructure, $\mathbf{v}_{\xi}$. Furthermore, we allow for a general velocity dispersion tensor $\boldsymbol{\sigma}_{\xi}$. We include the Heaviside function to truncate the velocity distribution at the escape speed. In practice, as the subcomponents are peaked away from $v_{\text {esc }}$ and as their velocity dispersion is relatively small, the effect of the truncation is negligible.

We make the approximation that the velocity dispersions $\boldsymbol{\sigma}_{\xi}$ that enter $f_{\xi}^{i}(\mathbf{v})$ are the same as the stellar components listed in Table II. This assumption is probably not true in detail. For instance, numerical simulations have shown that the DM stream associated with the Sagittarius stream are more extended and are misaligned from the stellar component [112, 113]. However, the progenitor of the Sagittarius stream is a dwarf irregular galaxy [114], which had its stars initially distributed in a disk and its DM in a round halo, so this mismatch is an extreme case. The dwarf spheroidals, which are believed to have produced S1, S2, and the other Shards [11], have DM and stellar populations that have similar spheroidal shapes before they were accreted. Nonetheless, the velocity dispersion of the DM and stars in the progenitor is not exactly the same, and so mismatches between DM and stellar streams are still expected, albeit on a smaller scale than for the Sagittarius stream. Our approximation is reasonable for the dwarf spheroidal regime, though work to fully test its accuracy using a numerical simulations is desirable.

\section{A. Weighting the halo components}

From Eq.(2), we also need to specify $\xi_{\mathrm{R}}, \xi_{\mathrm{S}}$ and $\xi_{i}$, the fractional contribution that each component makes to the local DM density. Although these cannot be determined from Gaia data, there are some general statements that we can make about their relative values. For example, the gravitational potential is nearly spherical [115], so the DM associated with the Sausage and the Shards is subdominant. In Ref. [72] it was argued that the Gaia Sausage can contribute around $20 \%$ to the local DM density without exceeding the bound of $1 \%$ on the ellipticity of the equipotentials of the Milky Way. A value $\sim 20 \%$ is also consistent with the fraction obtained from the FIRE2 simulations [71], and is in accordance with the interpretation of the Sausage's formation determined using the Auriga simulations [76, 116]. As the stellar Shards are not the dominant component of the main sequence stellar halo sample, it is unlikely that their progenitors will have brought more DM into the inner halo than, say, the Sausage. So $\xi_{\text {tot }}$ will certainly be smaller than $20 \%$.

To motivate us towards a lower limit to $\xi_{\text {tot }}$, we can refer to the library of N-body minor merger accretion events [117], as used in the interpretation of S1 and S2 in Ref. [11]. Streams originating from $\sim 10^{10} M_{\odot}$ and $\sim 5 \times 10^{9} M_{\odot}$ subhalos, believed to be the approximate total masses of the S1 and S2 progenitors, were found to contribute $\mathcal{O}(1 \%)$ to the local DM density at the solar location. The simulations made simplifying approximations about the DM distribution in the in-falling satellite and the host galaxy, and the impact of the Milky Way disk was not included. Despite these limitations, they suggest that a reasonable range for the total contribution from all of the substructures in Tables II and III is $1 \% \lesssim \xi_{\text {tot }} \lesssim 10 \%$. Following Ref. [72], we will fix $\xi_{\mathrm{S}}=20 \%$ and the round DM halo contributes the rest: $\xi_{\mathrm{R}}=80 \%-\xi_{\text {tot }}$.

After this, we are still left with the task of specifying $\xi_{i}$, the contribution from each individual Dark Shard. As discussed in Sec. III, to reflect the relative importance of the clusters of stars in action space, it is prudent to group the Shards into five categories: S1, S2, Retrograde, Prograde, and Low Energy components. We next apply an equal weighting to each category (e.g., each category contributes $2 \%$ when $\xi_{\text {tot }}=10 \%$ ). Finally, since the individual Shards within each category have different star counts and positions relative to the Solar System, we weight the contribution of the Shards within each category by the number of stars multiplied by the height of the spatial distribution of stars at the solar position.

While the weighting prescription just described is a benchmark case, we will see it captures much of the resulting phenomenology of the different categories of substructure. If slight changes to the weighting lead to particularly interesting experimental signals however, then these will also be discussed. 


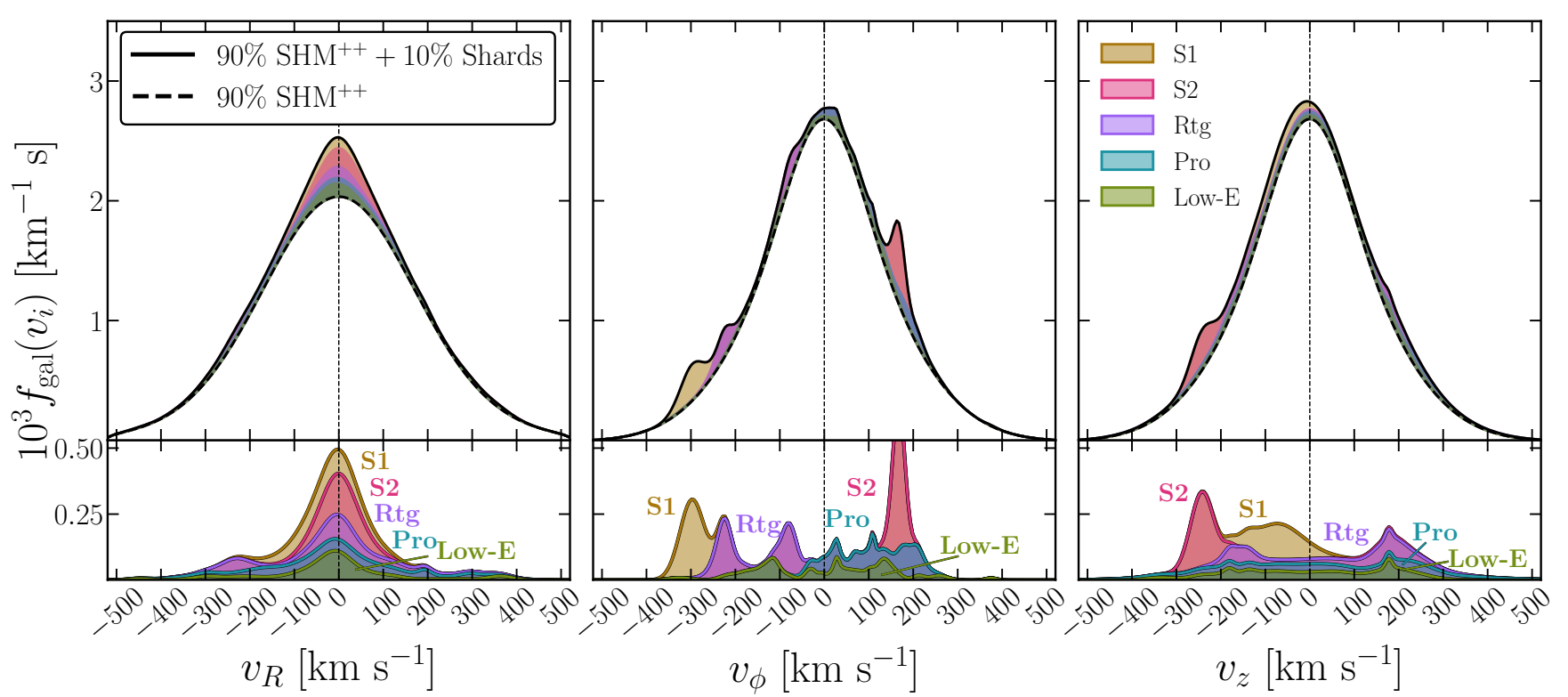

FIG. 5. The distributions of the three components of the galactic rest frame velocities. The solid black lines in the upper panels show the total distribution, including the isotropic Round Halo, Gaia Sausage and Dark Shard components assuming the Dark Shards contribute $10 \%$ to the local DM density $\left(\xi_{\text {tot }}=10 \%\right)$. The distribution without the Dark Shards is shown as the black dashed line. The coloured regions show the contribution from each of the S1, S2, Retrograde, Prograde, and Low Energy categories: these distributions are stacked one upon the other in reverse order to aid the visibility of all the features. The lower panels show the stacked distributions from the Dark Shards alone.

\section{B. The galactic rest frame distribution}

In Fig. 5 we present the galactic rest frame distributions of each galactocentric cylindrical polar velocity component. The upper solid black line shows the velocity distributions obtained when $\xi_{\text {tot }}=10 \%$ and the weighting prescription just described is used. The lower dashed black line shows the $\mathrm{SHM}^{++}$distribution normalised to $90 \%$. This is to highlight the effect of the $10 \%$ contribution from the Dark Shards which adds on top of the $90 \% \mathrm{SHM}^{++}$distribution: the five coloured regions between the dashed and solid black lines show the individual contribution from each of the S1, S2, Retrograde, Prograde, and Low Energy categories. The lower panels in Fig. 5 emphasise the range of velocities to which each category contributes. The enhanced radial anisotropy of the $\mathrm{SHM}^{++}$due to the Sausage is the reason that the $v_{R}$ distribution (left panel) is wider and shorter than the other distributions.

The S1 stream and the Retrograde Shards at large negative $v_{\phi}$ are interesting as they will become more prominent when boosted into the Earth's frame. Given that the Earth moves with $v_{\phi} \sim 250 \mathrm{~km} \mathrm{~s}^{-1}$, S1 and the Retrograde Shards blow into the Solar System with a large $v_{\phi} \sim 550 \mathrm{~km} \mathrm{~s}^{-1}$. Some of the phenomenology associated with the S1 stream has already been studied in Refs. [78, 102, 103].

The S2 steam and the Prograde Shards are interesting because they have a relatively large positive $v_{\phi} \sim$ $200 \mathrm{kms}^{-1}$ meaning they almost corotate with the
Earth. Corotating DM will have much lower speeds when boosted to the Earth's frame. While many methods of detecting DM are more sensitive to the high speed tail, axion haloscopes are notable because the speed distribution at low speeds can also give rise to distinctive experimental signals. Also important is the large negative $v_{z}$ of S2. This behaviour can be seen in Fig. 4 (middle right panel), which showed that S2 passes through the Milky Way disk from above. This means that S2, unlike S1, is highly misaligned from the expected orientation of the DM wind. It will therefore also be interesting from the perspective of directional and time dependent searches for DM.

\section{DARK MATTER SPEED DISTRIBUTION AND FLUX ON EARTH}

When observed on Earth, incoming DM particles are boosted from the galactic frame in which we have been working until now, into the lab frame. The lab-frame velocity distribution is

$$
f_{\text {lab }}(\mathbf{v})=f_{\text {gal }}\left(\mathbf{v}+\mathbf{v}_{\odot}+\mathbf{v}_{\oplus}\right)
$$

where $\mathbf{v}_{\odot}=\left(11.1, v_{0}+12.24,7.25\right) \mathrm{km} \mathrm{s}^{-1}$ is the sum of the local standard of rest and the peculiar velocity of the Sun [94, 118], and $\mathbf{v}_{\oplus}$ is the Earth's velocity around the Sun (we will neglect the Earth's daily rotation). We will include the time dependence of $\mathbf{v}_{\oplus}$ when we discuss modulation signals in Sec. VII A. Until that section, the 


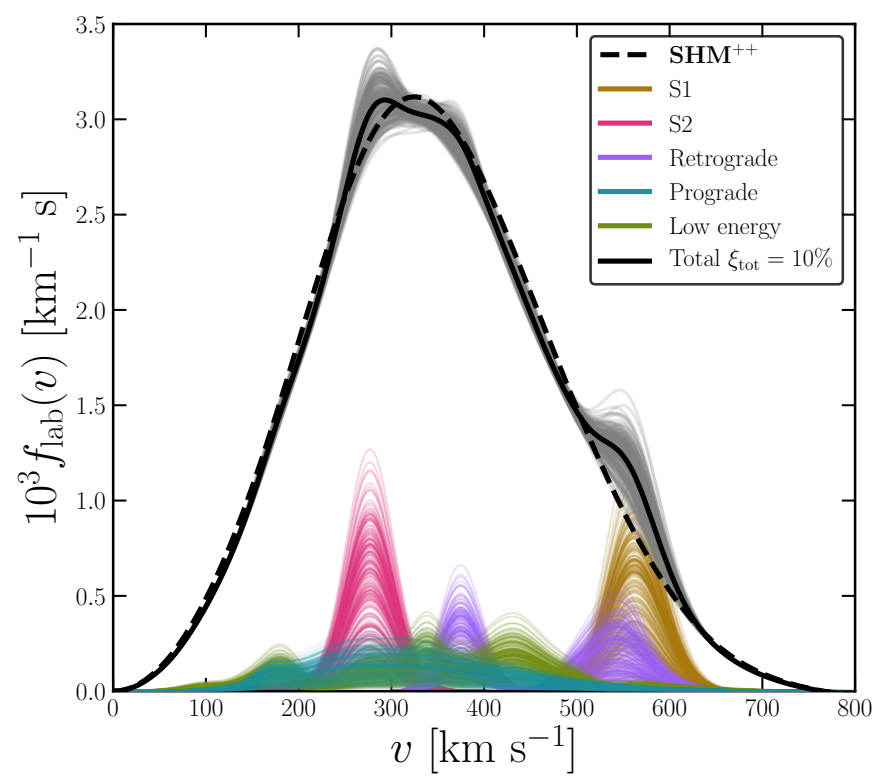

FIG. 6. The lab-frame DM speed distributions. The black solid line shows the distribution including the isotropic round halo, Gaia Sausage and Dark Shard components assuming $\xi_{\text {tot }}=10 \%$ and the weighting prescription described in Sec. IV A. For reference, the $\mathrm{SHM}^{++}$distribution is shown as a dashed black line. The grey and coloured lines show the total speed distribution and individual contribution from each category (S1, S2, Retrograde, Prograde and Low Energy) obtained when the weightings of each category are selected randomly (such that $\xi_{\text {tot }}=10 \%$ ). We see that the peak of the distribution (around $300 \mathrm{~km} \mathrm{~s}^{-1}$ ) can be changed by $\mathrm{S} 2$ and the Prograde category while the high speed tail can be modified by $\mathrm{S} 1$ or the Retrograde Shards.

time dependence of the Earth's motion will not impact our results, so we fix $\mathbf{v}_{\oplus}=(29.4,-0.11,5.90) \mathrm{kms}^{-1}$, the value on March 9, which gives a speed distribution equivalent to the time averaged result.

\section{A. Speed distribution}

Figure 6 shows the DM speed distribution in the lab frame. The black solid line shows the speed distribution, $f_{\text {lab }}(v)$, that is obtained when $\xi_{\text {tot }}=10 \%$ and the components are individually weighted according to the prescription described in Sec. IV A. The black dashed line shows the speed distribution from the $\mathrm{SHM}^{++}$model (equivalent to setting $\xi_{\text {tot }}=0$ ). The effect of S1, S2 and the Retrograde structures are particularly apparent, as they enhance the distribution around $280 \mathrm{~km} \mathrm{~s}^{-1}$ and $550 \mathrm{~km} \mathrm{~s}^{-1}$.

To explore the possible effects of different weightings, we have also shown the distributions that are obtained when the weights for the five substructure categories are drawn randomly from a uniform distribution, while still adding up to $\xi_{\text {tot }}=10 \%$. The effect on the overall speed distribution is shown by the thin grey lines in Fig. 6, while the contribution from the individual categories are shown by the coloured lines at the bottom of the figure. Some care should be taken when interpreting the envelope of the grey lines since, for example, if the contribution from $\mathrm{S} 2$ is maximal $\left(\xi_{\mathrm{S} 2}=10 \%\right)$ so that the peak at $\sim 280 \mathrm{kms}^{-1}$ takes the largest value, then there will be no peak at $\sim 550 \mathrm{~km} \mathrm{~s}^{-1}$ as the contribution from the other categories is zero. However, this exercise is useful as it highlights the general features that can emerge and that are captured by our benchmark weighting prescription; namely, the peak of the distribution (around $300 \mathrm{~km} \mathrm{~s}^{-1}$ ) can be changed depending on the weighting given to S2 and the Retrograde categories, and the high speed tail can be modified either by S1 or the Retrograde categories. The effects from the Prograde and Low Energy categories are less pronounced in comparison to S1, $\mathrm{S} 2$ and the Retrograde Dark Shards, but in general lead to a broad enhancement for speeds $v \lesssim 400 \mathrm{~km} \mathrm{~s}^{-1}$.

\section{B. Directional dependence of the flux}

In addition to the speed distribution, the directionality of the DM signal is important for many experiments. The prediction of an anisotropic flux of DM is a generic result that follows from our motion through the (essentially) non-rotating DM halo. For the simplest models in which the velocity ellipsoid (in the galactic frame) is centred at $\mathbf{v}=\mathbf{0}$ and there is no overall rotation, the DM flux on Earth peaks in the direction $-\mathbf{v}_{\text {lab }}$. This holds for both the isotropic part of the halo and the Sausage, so the flux is largest in the direction $-\mathbf{v}_{\text {lab }}$ [72], towards the constellation Cygnus. For substructures, this need not be the case. Since they may be characterised by nonzero average velocity in the rest frame of the Galaxy [cf. Eq.(8)], their lab-frame distribution will not necessarily align with Cygnus.

Figure 7 shows the angular distribution of the DM flux as seen on Earth. This is calculated by integrating along the line of sight $\hat{\mathbf{x}}_{\text {los }}$,

$$
\frac{\mathrm{d} \Phi_{\mathrm{DM}}}{\mathrm{d} \Omega_{\mathrm{los}}}=\rho_{0} \int_{0}^{\infty} v^{3} f_{\text {lab }}\left(-v \hat{\mathbf{x}}_{\mathrm{los}}\right) \mathrm{d} v,
$$

where $\rho_{0}=0.55 \mathrm{GeV} / \mathrm{cm}^{3}$ [72] is the local DM density. For this result and several others, we display angular distributions in terms of galactic longitude and latitude, $(l, b)$, which are mapped with a Mollweide projection.

The left panel shows the flux for the $\mathrm{SHM}^{++}$with no substructure (i.e., $\xi_{\text {tot }}=0$ ). The distribution is smooth and peaks towards Cygnus, but the contours of equal flux are not quite symmetric owing to the anisotropy brought by the Sausage. The right panel shows the flux when the Dark Shards contribute $\xi_{\text {tot }}=10 \%$ of $\rho_{0}$. Compared to the left panel, we see that the peak of the flux is now offset slightly from the direction of Cygnus, the maximum flux is much higher, and there are multiple peaks rather than one, with the most noticeable one appearing towards high galactic latitudes, $b \approx+70^{\circ}$. 

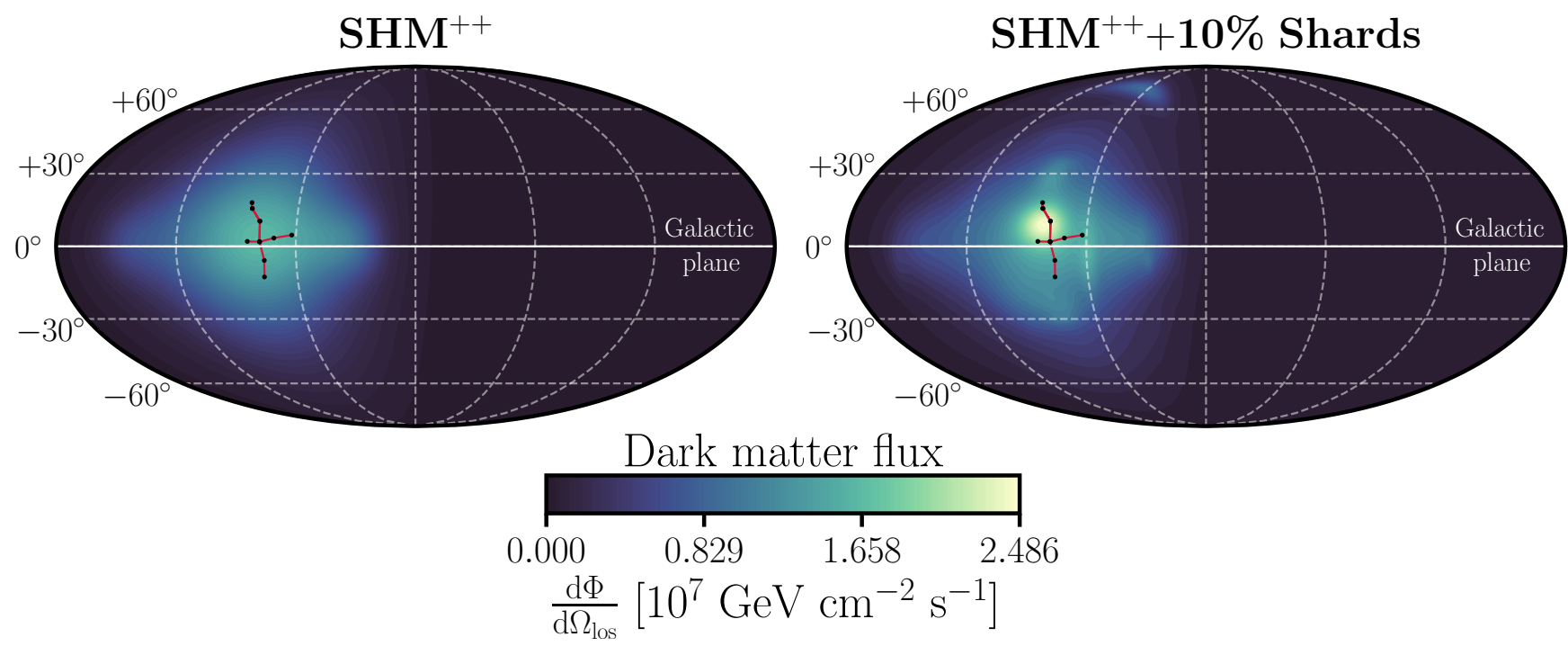

FIG. 7. Angular dependence of the DM flux on Earth, in terms of galactic longitude and latitude. The galactic plane is shown by the white horizontal line and the Cygnus constellation in red. The left panel shows the flux for the $\mathrm{SHM}^{++}$, while the right panel shows the flux when the Dark Shards contribute $\xi_{\text {tot }}=10 \%$. In contrast to the $\mathrm{SHM}^{++}$, which peaks in the direction of Cygnus and has a smooth distribution, the effect of the Dark Shards on the distribution is to enhance and slightly offset the peak towards Cygnus and to generate multiple subsidiary peaks.

To understand the origin of these differences, it is helpful to view the angular distributions of the individual categories. These are shown in Fig. 8, where we have drawn the $68 \%$ and $95 \%$ contours around each category's line-ofsight flux distribution. The S1 stream almost aligns with Cygnus because of its relatively small $v_{R}$ and $v_{z}$ (cf. Table II). The Retrograde Shards are also focused more strongly around Cygnus as the boost into the Earthframe increases the size of their $v_{\phi}$ components relative to $v_{R}$ and $v_{z}$. This is reversed in the case of the Prograde category, whose flux becomes spread over much wider angles relative to Cygnus. Finally, the prominent high latitude feature from Fig. 7 can clearly now be identified with S2. This should not be surprising given the large negative $v_{z}$ component that we observed in Fig. 5, indicating the presence of a component incoming from high latitudes. For visibility, we have not shown the Low Energy Shards which, similarly to the Prograde structures, are spread over much wider range of angles.

\section{AXION SEARCHES}

The changes we have made to the lab-frame speed distribution are relevant for all experiments searching for DM, but particularly axion searches. Since axions are so light, to make up the nonrelativistic galactic DM the axion field would need to have enormous, macroscopic occupation numbers per quantum state. As such the natural description of axionic DM is of an oscillating classical field, which for small enough time and length-scales can

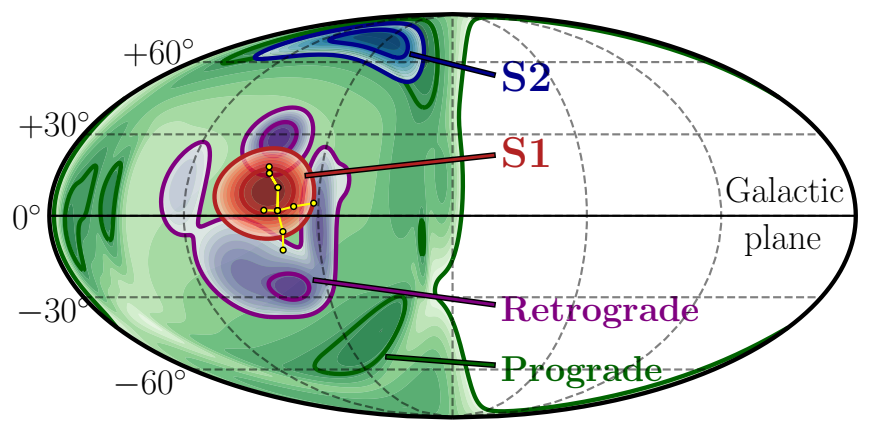

FIG. 8. Angular distribution of the Dark Shards (with the exception of the Low Energy category). For each component we delineate contours enclosing $68 \%$ and $95 \%$ of the flux distribution in angle. We see that S1 and the Retrograde Shards are focused around the Cygnus constellation, while the Prograde and S2 Shards are spread over much wider angles.

be written,

$$
a(t)=\frac{\sqrt{2 \rho_{a}}}{m_{a}} \cos \left[m_{a}\left(1+\frac{v^{2}}{2}\right) t-m_{a} \mathbf{v} \cdot \mathbf{x}+\phi\right],
$$

where $m_{a}$ is the axion mass, $\mathbf{x}$ is position, and $\phi$ is an arbitrary phase. The amplitudes of the oscillations of the axion field are expressed in terms of the axion density $\rho_{a}$, which is stochastically varying under an exponential distribution with mean $\rho_{0}$ (see e.g., Refs. [102, 119, 120] for further details).

The axion field is coherent according to Eq.(11) for timescales of the order $\tau_{a} \lesssim\left(m_{a} v^{2}\right)^{-1}$, where $v$ is a "typical" DM speed. The axion oscillations will decohere over timescales longer than $\tau_{a}$ and the subsequent tempo- 


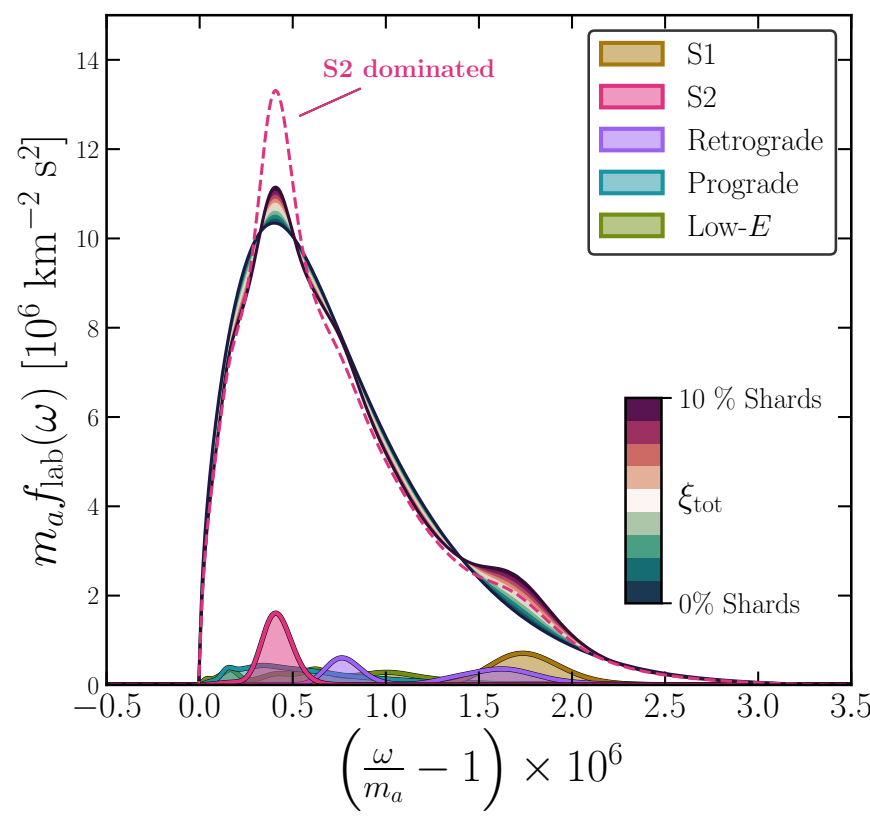

FIG. 9. The time-averaged spectral lineshape observable in an axion haloscope as a function of frequency $\omega$ relative to the axion mass $m_{a}$. The lineshape from the $\mathrm{SHM}^{++}+$Dark Shards equal weighting halo model is shown by the coloured region, from dark green to dark red corresponding to the range of values $0 \% \leq \xi_{\text {tot }} \leq 10 \%$. We also show the individual weighted contribution from each Shard category when $\xi_{\text {tot }}=10 \%$. S1 and the Retrograde Shards contribute to a bump at higher frequencies but $\mathrm{S} 2$ sharpens the peak of the distribution. To emphasise this, the pink dashed line shows the lineshape under an alternative weighting scheme in which half of $\xi_{\text {tot }}=10 \%$ is assigned to S2. This distribution is notably sharper and would enhance the sensitivity of axion haloscopes to the coupling $g_{a \gamma}$.

ral variation in frequency and phase will effectively map out the DM speed distribution when observed for long enough. Haloscope designs generically involve the monitoring of some electromagnetic response to $a(t)$. The spectral density of photons (usually the Fourier transform of time series data) measured over a time $t \gg \tau_{a}$ is proportional to $f_{\text {lab }}(v)$ up to stochastic variations. Hence the signal lineshape in axion haloscopes is very sensitive to the DM halo model.

The central problem in detecting the axion however is that we do not know the frequency, $\omega \simeq m_{a}(1+$ $\left.v^{2} / 2\right)$ at which the electromagnetic response to the axion field should be monitored. To search for this frequency, haloscopes either enforce a resonance or constructive interference condition for a signal oscillating at $\sim m_{a}$ (as in e.g., ADMX [121, 122], MADMAX [123, 124], HAYSTAC [125-128], CULTASK [129-131], ORGAN [132, 133], KLASH [134] and RADES [135]), or are sensitive to a wide bandwidth of frequencies simultaneously (e.g., ABRACADABRA [136-138], BEAST [139] and DM-Radio [140]). See Ref. [141] for a recent review.

For most of the haloscope techniques discussed, the spectral density of axion-induced photons is proportional to the speed distribution, up to a change of variables between frequency and speed (see e.g., Refs. [37, 142])

$$
\frac{\mathrm{d} P}{\mathrm{~d} \omega}=\pi \mathcal{H}(\omega) g_{a \gamma}^{2} \rho_{0} f_{\mathrm{lab}}(\omega),
$$

where $g_{a \gamma}$ is the axion-photon coupling on which the experiment will set a limit. We use $\mathcal{H}(\omega)$ to note that a haloscope will often have an underlying frequency dependence, but over the small width of the axion signal this is usually going to be flat. The relevant object for us is,

$$
f_{\text {lab }}(\omega)=\frac{\mathrm{d} v}{\mathrm{~d} \omega} f_{\text {lab }}(v) .
$$

We show $f_{\text {lab }}(\omega)$ as a function of frequency in Fig. 9. The coloured shading from dark green to dark red indicates the changes to the lineshape as $\xi_{\text {tot }}$ is increased from $0 \%$ (the $\mathrm{SHM}^{++}$) to $10 \%$. The contribution from the five Dark Shard categories when $\xi_{\text {tot }}=10 \%$ and the equal weighting scheme (cf. Sec. IV A) is shown at the bottom of the figure. We see similar effects to those shown in Fig. 6, but with an important difference: since $\omega \propto v^{2}$, the lineshape is more concentrated around low values than the speed distribution shown earlier. In fact, the peak itself is notably sharper relative to the $\mathrm{SHM}^{++}$ due to S2. However overall, the lineshape ends up being slightly wider due to the presence of S1 and the Retrograde Shards at higher frequencies. The effect from S1 was considered in detail in Ref. [78], including also the dependence on the S1 velocity dispersion.

In both resonant and broadband haloscopes, the sensitivity to $g_{a \gamma}$ is dependent upon how prominently the signal can show up over the experiment's noise floor. In a generic statistical methodology, this means that the sensitivity of an axion experiment scales as $g_{a \gamma} \sim$ $\left(\int f(\omega)^{2} \mathrm{~d} \omega\right)^{-1 / 4}$. Signals that are sharper in frequency are more prominent over white noise and hence easier to detect. Since the effect from S2 appears at the peak of the lineshape, if the local DM density had a larger contribution from S2, we would expect an even sharper lineshape. To demonstrate this explicitly, the pink dashed line in Fig. 9 shows the lineshape when half of $\xi_{\text {tot }}=10 \%$ is weighted towards S2 (as opposed to a fifth under the equal weighting scheme). This distribution is notably sharper and further increases the sensitivity of axion haloscopes to the coupling $g_{a \gamma}$.

We calculate a $2 \%-5 \%$ enhancement in the experimental sensitivity relative to the $\mathrm{SHM}^{++}$when the fraction of $\rho_{0}$ attributed to $\mathrm{S} 2$ is between $5 \%-10 \%$. The precise enhancement depends sensitively on S2's velocity dispersion, which - since there is a contamination from outlying stars - may be overestimated. To obtain more robust results from axion haloscopes, it is therefore important that the properties of $\mathrm{S} 2$ are characterised precisely. For instance, if the $\mathrm{S} 2$ velocity dispersion is reduced by $20 \%$ then we would expect a $10 \%-20 \%$ enhancement in axion sensitivity. Although this is a modest enhancement, as 
the axion mass scan rate to reach a given coupling scales as $g_{a \gamma}^{-4}$, it implies a reduction in the running times for reaching the sought-after DFSZ axion models [143, 144]. In fact the S2 enhancement is close to the enhancement brought about by the "N-body inspired" line shape often used in the presentation of results from ADMX (see e.g. Ref. [145]). This enhancement is modulo the increase in the local density between that model and the SHM.

Once the axion has been detected, it becomes more straightforward to measure the properties of streams. Since there will no longer be any need to scan the axion mass parameter, the experiment can reach a high statistics regime very rapidly and the signal will continue to amplify above the noise. For example, for ADMX to achieve DFSZ-sensitivity with signal-to-noise $S / N=5$ over a window of $20 \mu \mathrm{eV}$ in 5 years, around $\sim 10^{6}$ mass bins would be needed, corresponding to a typical time of $T \sim 100 \mathrm{~s}$ on each bin. To subsequently measure another peak in the spectrum which has an amplitude that was some fraction of the main signal, the noise would need to be reduced by that same ratio. Keeping the frequency resolution the same, the amplitude of white noise will scale with $1 / \sqrt{t}$. The S2 peak is around a fraction $0.23\left(\xi_{\mathrm{S} 2} / 5 \%\right)$ of the height of the main peak. Therefore we can estimate that around $(100 \mathrm{~s} / 0.23)^{2}=52$ hours would be required to measure $\mathrm{S} 2$ to the same $S / N$ as required to initially detect the DFSZ axion. From this measurement, the density of S2 relative to the rest of the halo can be determined from the height of the peak, as well as the lab frame speed and dispersion along one dimension from its central frequency and width. Furthermore, from the phase and amplitude of the annual modulation of S2, the rest of the 3-dimensional dispersion and velocity could be measurable on the timescale of a year. This procedure is discussed in much greater detail in Ref. [102]. A postdiscovery haloscope can be considered to be a very high-statistics instrument due to the alleviation of the need to scan over the axion mass.

\section{A. Dependence on the dark matter direction}

Some classes of axion experiments are also sensitive to the directionality of the DM flux. The CASPEr experiments for example [146] utilise spin-precession to detect the nuclear coupling of ultralight axions. The generic Hamiltonian that CASPEr is sensitive to has the form $\mathcal{H} \sim g \mathbf{I}_{N} \cdot \mathbf{D}$, where $\mathbf{I}_{N}$ represents the polarised nuclear spins and $\mathbf{D}$ is an effective field. The CASPEr-wind [147] experiment assumes that the effective field is given by the spatial gradient of the axion field,

$$
\mathbf{D}_{a}(t) \simeq-\sqrt{2 \rho(t)} \sin \left(m_{a} t+\phi\right) \mathbf{v}(t) .
$$

In this case, the Hamiltonian is proportional to the scalar product of the axion velocity and the polarised nuclear spin so the experiments are most sensitive when these two vectors are aligned. For higher mass axions (and

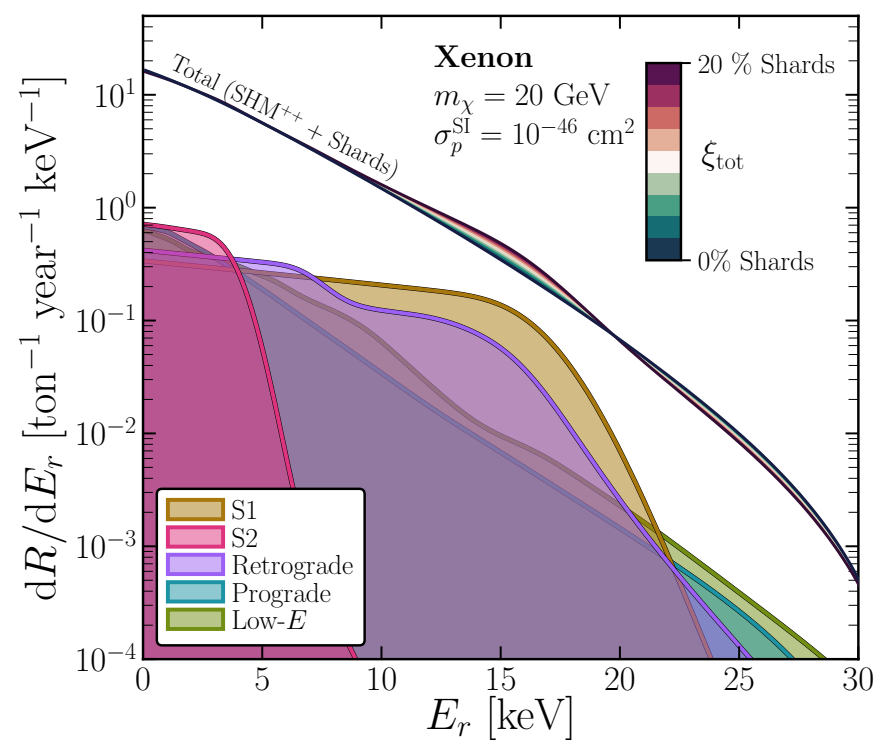

FIG. 10. Time-averaged differential event rate as a function of energy for a $20 \mathrm{GeV}$ WIMP-like particle scattering through a spin independent interaction with xenon. The coloured shading from green to red indicates how the event rate changes as the fraction $\xi_{\text {tot }}$ is increased from $0 \%$ to $20 \%$. The coloured lines at the bottom of the figure show the individual contribution from each category: S1, S2, Retrograde, Prograde and Low Energy. The dominant change in the spectrum comes from $\mathrm{S} 1$ around $15 \mathrm{keV}$, although the effect is always small, even when we increase the maximum limit of $\xi_{\text {tot }}$ to $20 \%$ to make the effect more noticeable.

electron and photon couplings), the ferromagnetic haloscope QUAX [148-150] also measures an effective field dependent on the axionic gradient.

One typically assumes a smooth DM flux that on average points in the direction of Cygnus $\hat{\mathbf{x}}_{\text {Cyg }}$. Yet we saw in Fig. 8 that one of the effects of the Dark Shards was to displace slightly the peak of the DM flux from the direction of Cygnus and to introduce a prominent high latitude component due to S2. This means that the gradient of the axion field as it varies over the coherence length and time will therefore be more likely to point at large angles away from Cygnus than under the assumption of the SHM. This will modify the daily modulation [102], and may potentially affect experimental sensitivities for CASPEr and QUAX. Similar arguments may also apply to experimental methods involving atomic clocks and comagnetometers that are searching for a wider class of ultralight particles [151-153]. We leave a more detailed investigation of this subject to future work.

\section{NUCLEAR RECOIL SIGNALS}

Many DM experiments currently operating search for signals from WIMP-like particles from the Milky Way halo scattering with nuclei through a wide variety of interactions [154-156]. For two-to-two scattering processes 
(which could be elastic or inelastic [157-160]), the timeaveraged rate $R$ of nuclear scatters as a function of the energy of the recoiling nucleus $E_{r}$ is found by integrating the flux of DM,

$$
\frac{\mathrm{d} R}{\mathrm{~d} E_{r}}=\frac{1}{m_{N}} \frac{\rho_{0}}{m_{\chi}} \int_{v>v_{\min }} v f_{\mathrm{lab}}(\mathbf{v}) \frac{\mathrm{d} \sigma_{T}\left(v, E_{r}\right)}{\mathrm{d} E_{r}} \mathrm{~d}^{3} v .
$$

Here, $m_{N}$ is the target nucleus mass, $m_{\chi}$ is the WIMPlike particle mass, $v_{\text {min }}$ is the minimum DM speed that can induce a recoil of energy $E_{r}$, and $\sigma_{T}$ is the DMnucleus scattering cross section, which can depend on $v$ and $E_{r}$.

It is commonplace to extract all factors of $v$ from the cross section and absorb them into the halo integral, a single object that encapsulates all of the velocity distribution dependence. For the canonical leading order spin independent (SI) and spin dependent (SD) DM-nucleus interactions, the differential cross section is inversely proportional to the square of the DM speed, $\mathrm{d} \sigma_{T} / \mathrm{d} E_{r} \propto v^{-2}$. In this case, the relevant halo integral is the mean inverse speed above $v_{\min }$,

$$
g\left(v_{\min }\right)=\int_{v>v_{\min }} \frac{f_{\mathrm{lab}}(\mathbf{v})}{v} \mathrm{~d}^{3} v .
$$

To show the impact of the Dark Shards on observable signals, we will consider the most familiar one: spin independent elastic scattering with equal couplings to protons and neutrons. In this case,

$$
\frac{\mathrm{d} \sigma_{T}\left(v, E_{r}\right)}{\mathrm{d} E_{r}}=\frac{m_{N} A^{2} \sigma_{p}^{\mathrm{SI}}}{2 \mu_{p}^{2} v^{2}} F^{2}\left(E_{r}\right) .
$$

Here, $A$ is the atomic number, $\mu_{p}$ is the DM-proton reduced mass, $F\left(E_{r}\right)$ is the nuclear form factor and $\sigma_{p}^{\mathrm{SI}}$ is the SI DM-proton scattering cross section. We display the differential event rate in Fig. 10 for an experiment with a xenon target (applicable for LZ [161], PandaX [162], XENON [163] and XMASS [164, 165]). The coloured shading from dark green to dark red indicates the changes to the differential rate as $\xi_{\text {tot }}$ is increased from $0 \%$ (the $\mathrm{SHM}^{++}$) to $20 \%$. The coloured regions at the bottom of Fig. 10 show the contribution from the individual Dark Shards. The S1 stream and most of the Retrograde Shards have the largest impact on the differential rate. $\mathrm{S} 2$ on the other hand, while prominent in other signals, is much less important here.

We find that the impact of the Dark Shards on $\mathrm{d} R / \mathrm{d} E_{r}$ is small, though this is not wholly unexpected. Xenon detectors, and nuclear recoil experiments in general, are poor at distinguishing features in the velocity distribution. Since only the recoil energy $E_{r}$ is measured while information about the recoil direction is lost, DM velocities cannot be reconstructed. Mathematically, this is encoded in the fact that the recoil rate is proportional to an integral over speeds, $g\left(v_{\min }\right)$, rather than the speed distribution explicitly as in the case of the axion-induced photon spectral density (cf. Eqs. (12) and (15)).

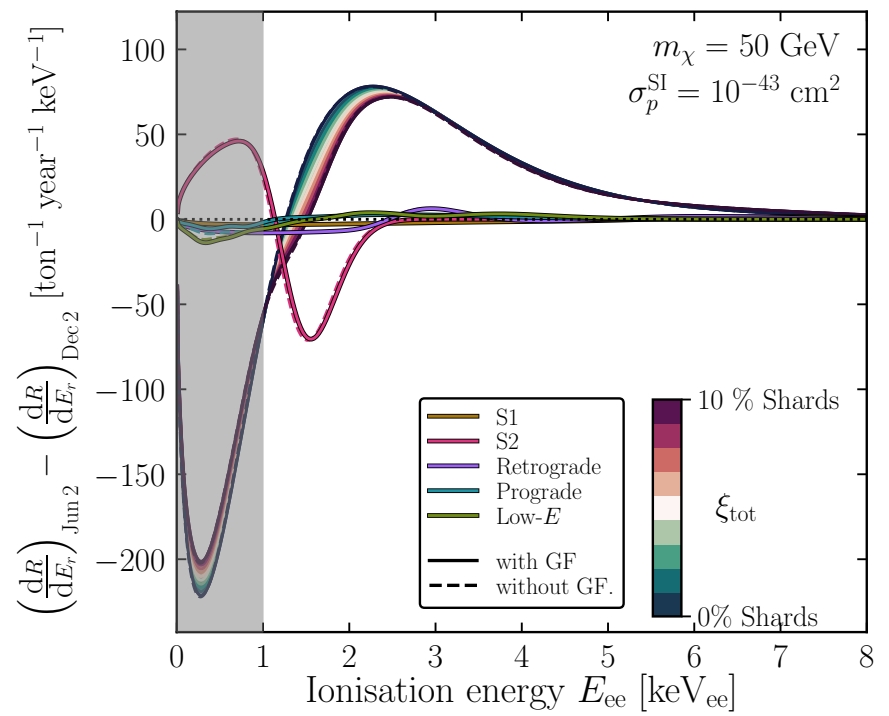

FIG. 11. Modulation amplitude (defined as the difference in the rate between June 2 and December 2) versus ionisation energy in a $\mathrm{NaI}$ scintillator for a $50 \mathrm{GeV}$ WIMP-like particle and a spin independent interaction. The shaded band indicates the change in the modulation amplitude as the value of $\xi_{\text {tot }}$ increases from 0 to $10 \%$ (green to red). The modulation amplitude decreases as $\xi_{\text {tot }}$ increases. The coloured lines show the modulation amplitude from the individual Dark Shards categories assuming (for visibility) that each contributes $20 \%$ of the local DM density. S2 is the most distinctive as its phase is reversed over most of this range of energies and its modulation amplitude is larger compared to the other Dark Shards. The dashed lines show the modulation amplitude when gravitational focusing is not included. They lie very close to the solid lines indicating that the effect on this measure of the modulation amplitude is small.

We displayed $\mathrm{d} R / \mathrm{d} E_{r}$ for a $20 \mathrm{GeV}$ WIMP-like particle here. For masses of this size scattering with xenon, the spectrum should in fact be the most sensitive to changes to $f(v)$ (see Ref. [78] for a quantitative discussion). However despite this choice, and even increasing the maximum value of $\xi_{\text {tot }}$ to $20 \%$ to make the changes more noticeable, they remain small.

As most of the changes in the recoil spectrum are brought about by S1, the phenomenology of the Dark Shards as a departure from the SHM will be very similar to the case studied in Ref. [78]. Following what was concluded then, we concur that the impact of the Dark Shards will be minor for nuclear recoil based experiments unless S1 and the Retrograde shards are particularly prominent in the DM of the ex situ halo.

\section{A. Modulation signals}

The results presented so far have been timeindependent. However, there are several classes of experiments for which the annual modulation brought about 


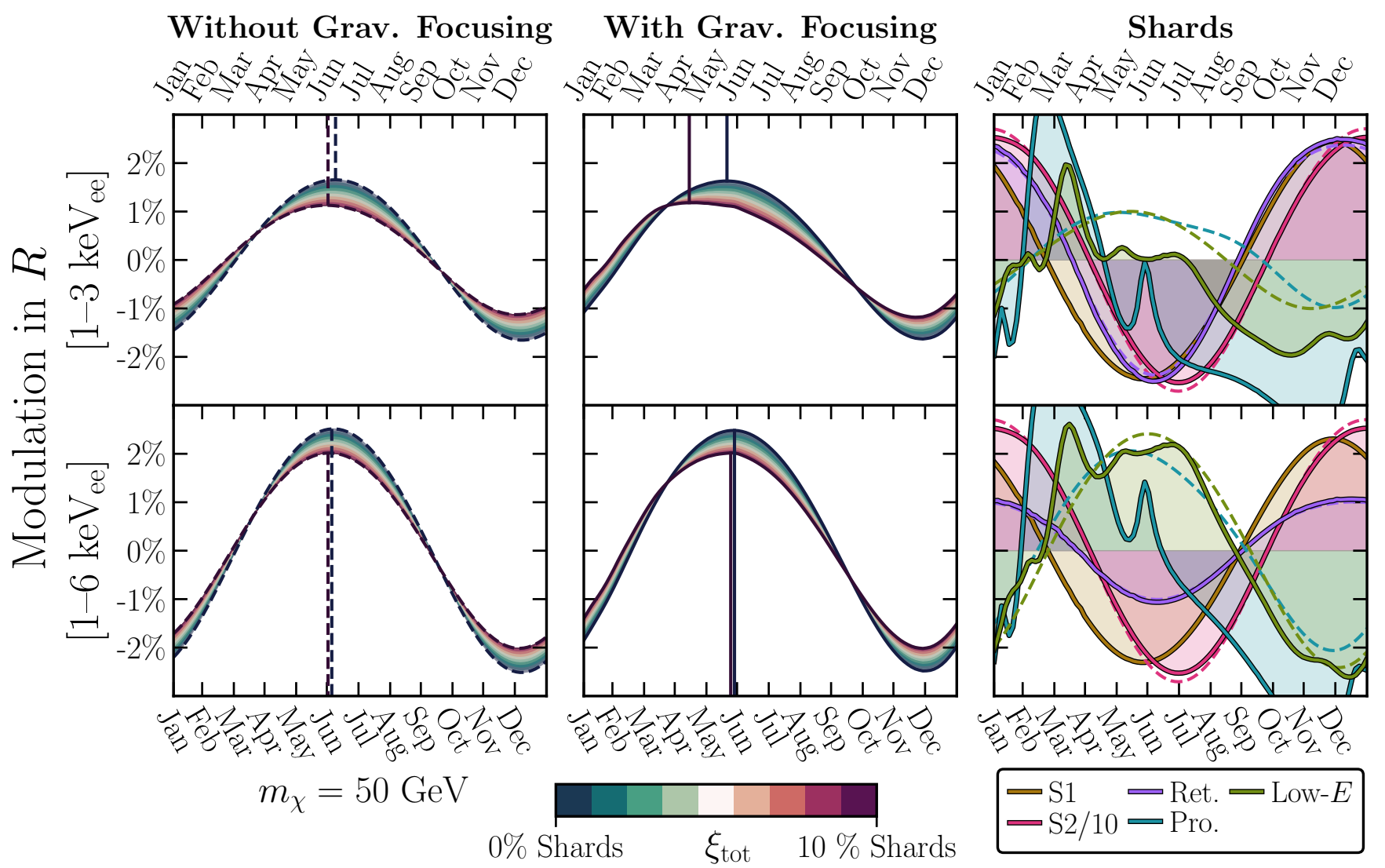

FIG. 12. The modulation of the integrated event rate in two ionisation energy bins of 1-3 and 1-6 keV (top and bottom panels in each column respectively). The WIMP particle model is kept the same from the previous Figure. The left-hand column shows the calculation ignoring gravitational focusing whereas the middle column shows the nonsinusoidal modifications that it gives rise to when it is included. The right-hand column shows each Dark Shard contribution individually. For visibility here we have suppressed the S2 modulation by a factor of 10 as it is by far the dominant contribution for this DM mass and for these energy bins.

by the Earth's motion around the Sun is the primary signal $[107,166]$. As the velocity distributions of the Dark Shards do not have an average velocity $\mathbf{v}=0$ in the galactic rest frame, they will distort modulation signals relative to the rest of the halo that does have zero average velocity.

To obtain the modulation signal for a detector in a laboratory on Earth, $f_{\text {lab }}(\mathbf{v}, t)$, two effects have to be taken into account. The first is due to the time dependence in the Earth velocity, which can be written as,

$$
\mathbf{v}_{\oplus}(t)=v_{\oplus}\left[\cos \left[\omega\left(t-t_{a}\right)\right] \hat{\boldsymbol{\epsilon}}_{1}+\sin \left[\omega\left(t-t_{a}\right)\right] \hat{\boldsymbol{\epsilon}}_{2}\right] .
$$

The Earth's velocity oscillates with frequency $\omega=$ $2 \pi /(1$ year $), t_{a} \simeq 22$ March is the Vernal Equinox, $v_{\oplus}=29.79 \mathrm{~km} \mathrm{~s}^{-1}$ is the average speed, and

$$
\begin{aligned}
& \hat{\boldsymbol{\epsilon}}_{1}=(0.9941,0.1088,0.0042), \\
& \hat{\boldsymbol{\epsilon}}_{2}=(-0.0504,0.4946,-0.8677),
\end{aligned}
$$

are orthogonal unit vectors for the Earth's orbital plane expressed in galactic coordinates. For brevity, we have suppressed the corrections from the eccentricity of the Earth's orbit, but they are included up to second order in our numerical results.

The second effect, known as gravitational focusing [167-172], takes into account the Sun's gravitational influence on DM particles in the Solar System. For particles blowing through the Solar System, the Sun acts as a gravitational lens that enhances the flux in March relative to half a year later in September [167, 170]. This effect is out of phase with the annual modulation induced by the Earth's rotation $\mathbf{v}_{\oplus}(t)$, which enhances the flux in June and reduces it in December [166]. Ultimately, gravitational focusing is negligible for time-averaged signals [169], however it can be an important modification to consider for experiments that make use of timing information [170].

When both effects are included, the time-dependent lab-frame velocity distribution is

$$
f_{\text {lab }}(\mathbf{v}, t)=f_{\text {gal }}\left(\mathbf{v}_{\odot}+\mathbf{v}_{\infty}\left[\mathbf{v}_{\oplus}(t)+\mathbf{v}\right]\right) .
$$

The function $\mathbf{v}_{\infty}[\mathbf{v}]$ gives the velocity that a particle would have had at infinity to have fallen into the So- 
lar System with the velocity v. Reference [169] showed that it can be expressed as

$$
\mathbf{v}_{\infty}[\mathbf{v}]=\frac{v_{\infty}^{2} \mathbf{v}+\frac{1}{2} v_{\infty} u_{\mathrm{esc}}^{2} \hat{\mathbf{x}}_{\oplus}(t)-v_{\infty} \mathbf{v}\left(\mathbf{v} \cdot \hat{\mathbf{x}}_{\oplus}(t)\right)}{v_{\infty}^{2}+\frac{1}{2} u_{\mathrm{esc}}^{2}-v_{\infty}\left(\mathbf{v} \cdot \hat{\mathbf{x}}_{\oplus}(t)\right)},
$$

where $u_{\text {esc }}=\sqrt{2 G M_{\odot} / r_{\oplus}(t)} \simeq 42.3 \mathrm{~km} \mathrm{~s}^{-1}, v_{\infty}^{2}=|\mathbf{v}|^{2}-$ $u_{\mathrm{esc}}^{2}$ and $\hat{\mathbf{x}}_{\oplus}(t)=-\sin \left[\omega\left(t-t_{a}\right)\right] \hat{\boldsymbol{\epsilon}}_{1}+\cos \left[\omega\left(t-t_{a}\right)\right] \hat{\boldsymbol{\epsilon}}_{2}$. In the limit that $M_{\odot} \rightarrow 0, \mathbf{v}_{\infty}[\mathbf{v}] \rightarrow \mathbf{v}$ and we recover the usual result in the absence of gravitational focusing.

To highlight the impact of the Dark Shards on modulation signals, we focus on the best known experiments searching for it; namely DAMA/LIBRA [173, 174], together with the experiments trying to replicate DAMA/LIBRA's result by employing similar technology and $\mathrm{NaI}(\mathrm{Tl})$ crystals. These include DMIce [175], KIMS [176], SABRE [177], Cosine [178, 179] and ANAIS [180,181]. We do not provide a detailed fit to the DAMA/LIBRA data but instead focus our discussion on the general features introduced by the Dark Shards.

For more than a decade, the DAMA/LIBRA experiment has measured a persistent annually modulating signal above known backgrounds (however, see Refs. $[182,183])$ with a significance in excess of $9 \sigma$ [174]. Although there is difficulty in explaining this signal with galactic DM while remaining consistent with other experiments, the signal does have many of the properties expected from galactic DM. ANAIS $[180,181]$ and Cosine $[178,179]$ have released first results and do not observe a statistically significant modulation, but they still require a few years of exposure to definitively test the DAMA/LIBRA result.

To calculate the observable modulation signal for DAMA/LIBRA and the other $\mathrm{NaI}(\mathrm{Tl})$-based experiments, we need to convert from the recoil energy $E_{r}$ to the ionisation energy $E_{\mathrm{ee}}$. They are related by $E_{\mathrm{ee}}=$ $Q E_{r}$, where $Q$ is the quenching factor. DAMA/LIBRA have historically used the values $Q=0.3$ and 0.09 for $\mathrm{Na}$ and I respectively [184], but we use the lower value $Q=0.1$ for Na to reflect more recent measurements [185]. To account for the finite PMT resolution, we convolve the rate by a Gaussian energy-dependent energy resolution with width, $\sigma_{\mathrm{E}}\left(E_{\mathrm{ee}}\right)=\alpha \sqrt{E_{\mathrm{ee}}}+\beta E_{\mathrm{ee}}$, where $\alpha=0.448 \sqrt{\mathrm{keV}_{\mathrm{ee}}}$ and $\beta=9.1 \times 10^{-3}$.

In Fig. 11, we show one particular measure of modulation amplitude as a function of ionisation energy for a $50 \mathrm{GeV}$ particle. The modulation amplitude is calculated by taking the difference of the differential recoil event rate on June 2 and Dec 2 (the days when the differential rate is maximised/minimised in the Standard Halo Model). This amplitude exhibits the well-known phase flip, here at $E_{\mathrm{ee}} \approx 1.3 \mathrm{keV}_{\mathrm{ee}}$. As in previous figures, the effect of the Dark Shards is indicated by the shading in Fig. 11. We see that as $\xi_{\text {tot }}$ increases (i.e., moves from green to red), the amplitude of the modulation amplitude decreases in the window between 1 and $3 \mathrm{keV}_{\mathrm{ee}}$ and in the grey shaded region below $1 \mathrm{keV}_{\text {ee }}$ (which is below the energy threshold set for DAMA/LIBRA phase-2).
The coloured lines in Fig. 11 show the modulation amplitude for the individual Dark Shard categories. The coloured lines make it clear that S2 leads to the most significant change. S2 has a large modulation amplitude and the phase flip is reversed relative to the other Dark Shards and the smooth components of the halo: for S2 the rate in June is smaller at low energies while the rate in December is larger at higher energies. The dominant direction of S2 is towards negative galactic- $Z$ (cf. Fig. 4) so the scattering rate at higher energies will be largest when the Earth's velocity is moving fastest in positive $Z$. For the Earth's orbit, this occurs in December, explaining the behaviour in Fig. 11. None of the other Dark Shards move on orbits so distinct from the smooth halo component (cf. Fig. 7), which is why their modulation behaviour is similar to the smooth halo component.

The dashed and solid lines in Fig. 11 show the modulation amplitude without and with gravitational focusing. The dashed and solid lines largely overlap showing that the effect of focusing on this measure of the modulation is small.

To make the impact of gravitational focusing more apparent, we show in Fig. 12 the time-dependent component of the rate over the course of one year for the same DM mass and cross section used in Fig. 11. The rate versus time in Fig. 12 has been integrated over two recoil energy bins of 1-3 and 1-6 $\mathrm{keV}_{\mathrm{ee}}$ (aligning with results often presented by DAMA/LIBRA). The left-most panels show the rates without focusing, which are clean sinusoidals. The main effect of the Dark Shards is to reduce the modulation amplitude, whereas the day when the rate is maximum (indicated by the dashed vertical lines) is changed only slightly. The middle panels show the rates when gravitational focusing is included. For the 1-3 $\mathrm{keV}_{\text {ee }}$ bin in particular, the effect is large: the peak day shifts by more than one month and the sinusoid is distorted. The right panels show the effects from the individual Dark Shards. The dominant component causing this change is S2 (in fact the modulation of S2 has been suppressed by a factor of ten in the right-hand column of Fig. 12 so that all categories are visible). The structure of $\mathrm{S} 2$ is disjoint in velocity space, with a prominent wrap at negative vertical velocity and a subsidiary wrap at positive velocity. This is also discernible in the middle panel of Fig. 4. The gravitational focusing of the annual modulation signal is particularly affected by the orientation of the subsidiary wrap, (S2b in Table II) which roughly lies in the plane of the ecliptic. This is an intriguing result, however some doubt has been cast in the literature [77] as to whether this additional wrap is truly a component of S2, or merely a collection of stars lying at a coinciding with S2 in action space. Ongoing work will address this issue in the near-future.

In light of this result, we reiterate the conclusion of Sec. VI that the S2 stream is surprisingly important for some DM signals and deserves to be studied in much greater depth. Furthermore, the comparison of Figs. 12 and 11 inspires us to urge that data from annual modula- 


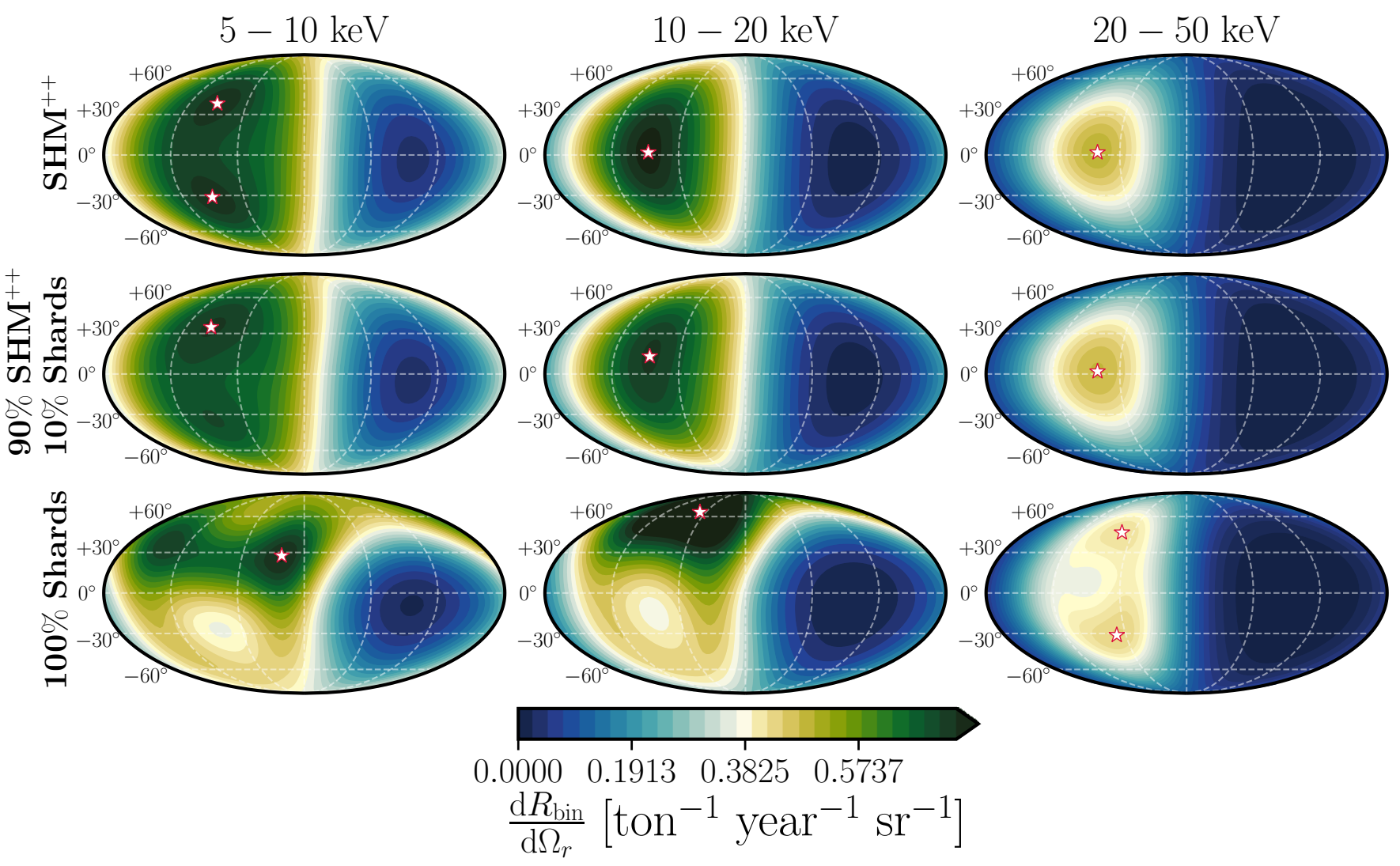

FIG. 13. Mollweide projection in galactic coordinates of the value of the angular recoil rate, integrated over the recoil energy bins displayed at the top of the figure. We assume a $100 \mathrm{GeV} \mathrm{DM}$ mass with $\sigma_{p}^{\mathrm{SI}}=10^{-46} \mathrm{~cm}^{2}$ scattering with ${ }^{19} \mathrm{~F}$. The upper three panels show the angular distribution of the $\mathrm{SHM}^{++}$, the middle panels show the distribution after the inclusion of a $10 \%$ contribution from the Dark Shards and the lower panels show just the Shards distribution (equivalent to $\xi_{\text {tot }}=1$ ). In each case we account for an angular resolution of $20^{\circ}$. The peak direction or directions are indicated with white stars.

tion searches be analysed in a framework that allows for higher harmonics (this requires of course that energy and timing information on recoil events are made available). Many of the interesting effects displayed in Fig. 12 are missed when calculating a modulation amplitude which assumes a single sinusoid with a fixed peak day. Whereas they could be picked up by a Fourier mode decomposition. We have left an exercise in such a calculation in the GitHub repository attached to this paper [79], but in the interest of keeping this section concise we do not discuss this further.

\section{B. Directional signals}

We showed in Fig. 7 that the angular dependence of the DM flux exhibits marked differences in our model compared with the expectation from a smooth halo. Many classes of experiment searching for DM are sensitive to this directional dependence of the underlying signal, as already discussed in the context of axions in Sec. VI A. Now we turn to the directional dependence of nuclear recoil signals.
The detection of the directionality of nuclear recoils is a promising technique for the discovery of WIMP-like particles [186]. The galactic anisotropy of the DM flux cannot be mimicked by any known terrestrial [187] or cosmic background [188], including solar neutrinos [189, 190]. However, measuring the recoil direction for $\mathrm{keV}$-scale recoil tracks is experimentally challenging. In liquid or solid state detectors, recoil tracks are typically $\mathcal{O}(\mathrm{nm})$, whereas in gas they can be $\mathcal{O}(\mathrm{mm})$. This means that a directional detector requires either a readout method with high spatial resolution (such as x-ray imaging of nuclear emulsions $[191,192]$ ) or detection media with very low pressure. We focus on the latter technique here since low-pressure gas time projection chambers (TPCs) have seen many more years of consistent progress in their experimental realisation, see e.g., Refs. [193-201]). Most recently the design for a tonne-scale network of directional gas-TPCs named CYGNus [202] has been put forward. The preliminary design study for CyGNUS is for a gaseous time projection chamber with a total active volume between $1000 \mathrm{~m}^{3}$ and $100,000 \mathrm{~m}^{3}$, using a He:SF 6 gas mixture at atmospheric pressure [202].

Directional nuclear recoil detectors such as CyGnus 

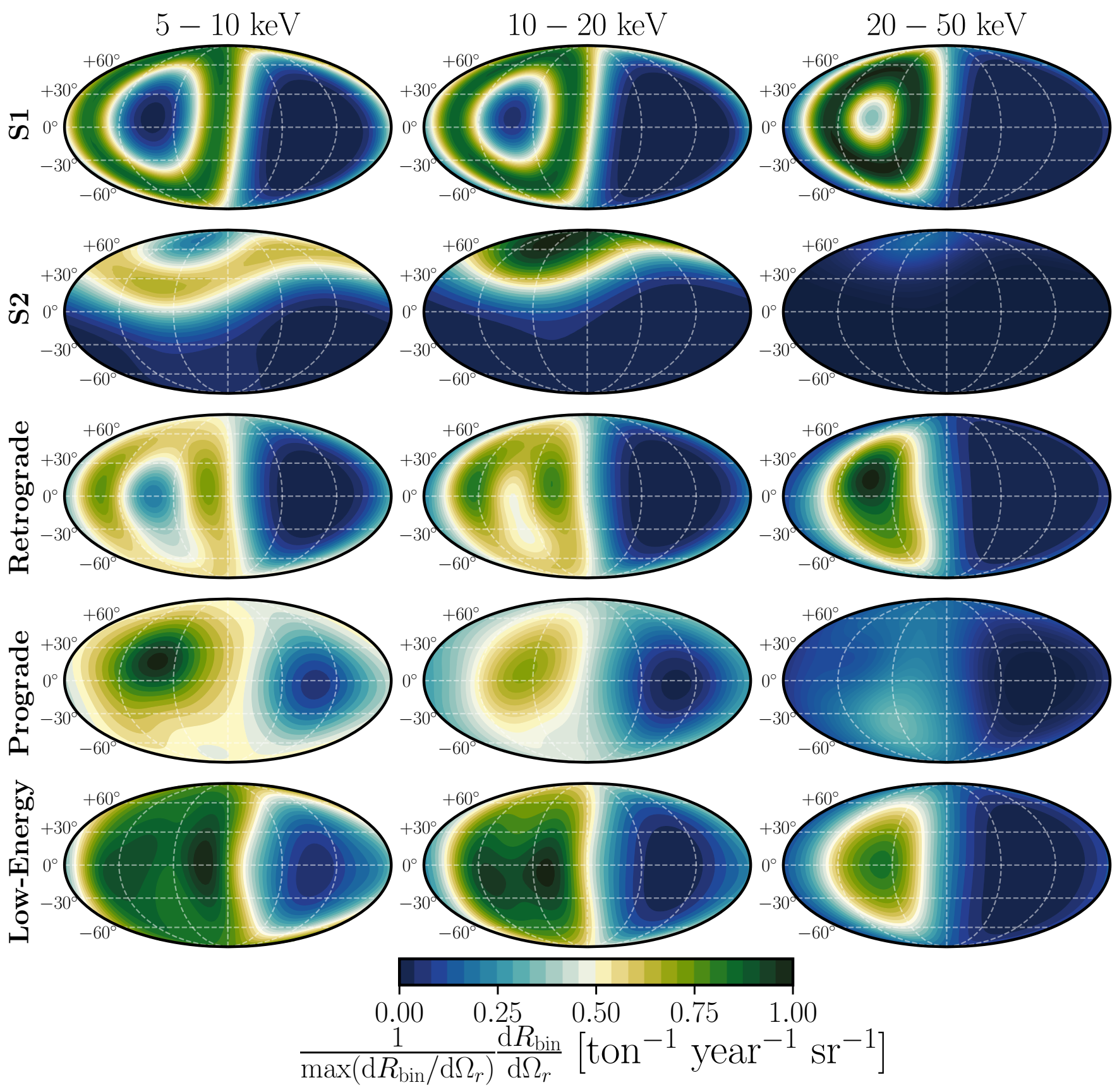

FIG. 14. As in the bottom row of Fig. 13 but with each Shard category shown individually. Since S1 and S2 are streams focused around single directions, their recoil distributions appear as rings with recoil energy-dependent angular radii. The Prograde, Retrograde and Low Energy categories have multiple components so lead to more complex topologies. Lower energy recoils are associated with larger angles away from Cygnus; recoil directions from Low Energy and Prograde Shards in particular extend much more widely over the sky.

aim to measure the direction $\hat{\mathbf{q}}$ associated with each nuclear recoil in addition to the nuclear recoil energy $E_{r}$. The double differential event rate for spin independent elastic scattering as a function of recoil energy, recoil di- rection and time is,

$$
\frac{\mathrm{d}^{2} R(t)}{\mathrm{d} E_{r} \mathrm{~d} \Omega_{r}}=\frac{1}{2 \pi} \frac{\rho_{0}}{m_{\chi}} \frac{A^{2} \sigma_{p}^{\mathrm{SI}}}{2 \mu_{p}^{2}} F^{2}\left(E_{r}\right) \hat{f}\left(v_{\min }, \hat{\mathbf{q}}, t\right) .
$$

This formula is similar to the non-directional rate for spin independent scattering, except for the factors of $1 / 2 \pi$ and $\hat{f}\left(v_{\min }, \hat{\mathbf{q}}, t\right)$ instead of $g\left(v_{\min }, t\right)$. This is the "Radon 
transform" of the velocity distribution [203, 204]:

$$
\hat{f}\left(v_{\min }, \hat{\mathbf{q}}, t\right)=\int \delta\left(\mathbf{v} \cdot \hat{\mathbf{q}}-v_{\min }\right) f_{\mathrm{lab}}(\mathbf{v}, t) \mathrm{d}^{3} v .
$$

We show the angular recoil distributions integrated over three recoil energies bins in Fig. 13. We assume spin independent scattering with $m_{\chi}=100 \mathrm{GeV}$ and a fluorine target, and apply a Gaussian angular resolution of $20^{\circ}$. We compare the recoil maps from the $\mathrm{SHM}^{++}$which contains only the smooth components of the halo (top row), the halo model with Dark Shards where $\xi_{\text {tot }}=10 \%$ (middle row), and the recoil maps from the Dark Shards alone (bottom row). Figure 14 can be used to observe each of the categories individually for comparison.

Over all energy bins in Fig. 13, the rate peaks in the forward half of the sky. One interesting and prominent feature in the top row (where $\xi_{\text {tot }}=0$ ) is that the rate has two maxima at positive and negative galactic latitudes of $\pm 60^{\circ}$. This is due to the influence of the radially anisotropic Sausage. The intuition behind this feature is subtle, but can be thought of as a manifestation of the ring feature originally discussed in the context of directional detectors in Ref. [205]. The ring feature shows up at low recoil energies for large DM masses and isotropic halo models. In a certain range of low energies, the Radon transform is not maximised at $\hat{\mathbf{q}}=-\hat{\mathbf{v}}_{\text {lab }}$ as it is at high energies, but rather at an energy-dependent angle away from $\mathbf{v}_{\text {lab }}$. This leads to recoils being focused along a ring around $\mathbf{v}_{\text {lab }}$. For the radially anisotropic Sausage there are instead two rings centred at wide angles away from $\mathbf{v}_{\text {lab }}$. The centres of the rings are closer to the galactic radial axis but do not precisely align with it because of the boost into the lab frame. When we calculate the recoil map for the Sausage across the sky, the rate is maximised when the two rings intersect. Because the ring centres are equal angular distances away from $\mathbf{v}_{\text {lab }}$ in galactic longitude, the intersections occur at galactic latitudes above and below $\mathbf{v}_{\text {lab }}$, hence the position of the two stars in the top panel of Fig. 13.

The influence of the Shards when included as a part of this distribution is to distort the symmetry of these two maxima about the galactic plane. The Shards also present several interesting effects when comparing the distribution across the sky between bins in recoil energy. Each Shard presents its own ring feature centred around the direction of its velocity. Kinematically a nuclear recoil will scatter at smaller angles away from the direction of the impinging particle, and in this context we see that as the angular radii of each ring becoming smaller as recoil energy increases. This can be seen in the panels for the S1 and S2 streams in Fig. 14.

The impact of the Shards is notable departures from the central assumption in directional detection, which is that the flux is rotationally symmetric around the Cygnus constellation. The overall forward-backward anisotropy is however largely preserve, despite the fact that some of the Low Energy and Prograde categories have sizeable event rates in the backward half of the sky (see Fig. 14). If the anisotropy were reduced, this could harm the powerful discovery capabilities of directional WIMP searches [186], so it is comforting that this is not the case. The anisotropy in fact seems to be quite strongly peaked in the forward direction when we go to the higher energy bins. This is largely because the Shards that have the largest angles away from Cygnus do not have recoils scattering at higher energies. It is only the retrograde substructures that align with Cygnus that are still present at high recoil energies and therefore add to the anisotropy.

The sum of all the Dark Shards would likely require many hundreds of events to distinguish from a smooth DM halo distribution. Nevertheless, in the context of WIMP-like dark matter, the measurement of the directionality of nuclear recoils would likely be the only feasible way to probe this part of the DM halo directly. Fewer events would be required in the cases of single streams such as S1 and S2, which lead to much more striking changes to the expected directional signals. To quantify these statements, we can estimate the number of events required in a benchmark directional detector to measure a $3 \sigma$ dipole anisotropy in the rates, $\mathcal{A}_{12}=R_{1} / R_{2}$, between two hemispheres, labelled "1" and "2":

$$
N_{\text {aniso }} \approx\left(3 \frac{R_{1}+R_{2}}{R_{1}-R_{2}}\right)^{2} .
$$

The formula arises from simply assuming that the contrast in the rates, proportional to $\left(R_{1}-R_{2}\right)$, between the two hemispheres should be greater than a $3 \sigma$ deviation in the total number, proportional to $3 \sqrt{R_{1}+R_{2}}$, in order to measure the anisotropy.

We assume two orthogonal pairs of hemispheres, a "forward-backward" pair, which align with $\mathbf{v}_{\text {lab }}$, and an "up-down" pair, which is aligned $90^{\circ}$ from the forwardbackward hemispheres, and approximately align with the Galactic North Pole. Taking a typical directional detector model assuming ${ }^{19} \mathrm{~F}$ recoils above a threshold of $3 \mathrm{keV}$, under the $\mathrm{SHM}^{++}$the forward-backward anisotropy is on the order of $\mathcal{A}_{\mathrm{fw}} \sim 5$ (requiring around 20 events to detect) and there is no up-down anisotropy, so $\mathcal{A}_{\mathrm{ud}}=1$. We find that over all detectable values of the DM mass, the Dark Shards in total give a negligible change to both $\mathcal{A}_{\mathrm{fw}}$ and $\mathcal{A}_{\mathrm{ud}}$. So the forward-backward dipole is largely preserved, but the slight up-down asymmetry caused by the Dark Shards would need in excess of $N_{\text {aniso }} \simeq 1000$ events for $\xi_{\text {tot }} \lesssim 50 \%$.

Isolating $\mathrm{S} 1$ and $\mathrm{S} 2$, we find that they give rise to opposing effects, which reflect their perpendicular velocities. S1 enhances the forward-backward anisotropy, approximately linearly with $\xi_{\mathrm{S} 1}$, whereas $\mathrm{S} 2$ enhances the up-down anisotropy with $\xi_{\mathrm{S} 2}$. For $10 \%$ of the local density in $\mathrm{S} 1$, we find that $\mathcal{A}_{\mathrm{fw}}$ is enhanced such that it would reduce the required number of events to detect it from 20 to $17 ; \mathcal{A}_{\mathrm{ud}}$ is essentially unchanged. For $10 \%$ in $\mathrm{S} 2$, the required number of events to detect $\mathcal{A}_{\mathrm{fw}}$ is only mildly increased from 20 to 23 , but the up-down anisotropy is increased dramatically and is detectable with $N_{\text {aniso }} \simeq 250$ 
events as opposed to 1000. While these numbers are large, it is worth pointing out that in some ways they reflect upper limits. This simple procedure of calculating dipole anisotropies along two directions represents a model-independent test that could be performed on directional data but it does not optimise for the particular direction of a given stream, or include the correlation with recoil energy information. A full likelihood-based analysis that includes this additional information would therefore require fewer events than we have estimated here.

\section{SUMMARY}

Thanks to Gaia, the six-dimensional view of our local stellar halo is more extensive than ever before. The distributions of stars in action space are revealing much about the recent accretion history of the Milky Way. We have studied the properties of the substructure found in this distribution due to accretion during the ex situ phase of the Milky Way's formation. Substructure passing close by the Earth has implications for a wide range of experiments looking for DM.

The sample of stars we study here is derived from a cross-matching of the SDSS and Gaia catalogues. Taking inspiration from the kinematic structure of the stellar halo in this sample, we began by describing the two smooth components present in the dark halo: a roundish, isotropic part and a radially anisotropic "sausage"shaped part. To this, we then introduced substructures. We considered in particular the large collection of action space substructures, or "Shards", present in the GaiaSDSS stellar halo sample. We individually modelled the Shards, identified those passing closest to the solar neighbourhood, and then used the models to build an approximate description for the collection of their DM counterparts: the "Dark Shards". We organised the Dark Shards into five categories in terms of overall significance and properties: S1, S2, Retrograde, Prograde, and Low Energy. When accounting for the Dark Shards, the resulting DM velocity and speed distributions contain notable nonGaussian departures from their conventionally assumed Gaussian and Maxwellian forms (see Figs. 5 and 6).

Of the long list of Shards, we highlight the S1 and S2 streams as the most important ones nearby. They are present at highest significance in action space [12, 26] and have the most important experimental implications. S1 has a very high Earth-frame speed of $\sim 550 \mathrm{~km} \mathrm{~s}^{-1}$, so is important for signals which are sensitive to the tail of the speed distribution. Several other substructures on retrograde orbits lead to phenomenologically similar effects. These may be observable in nuclear recoil searches for WIMPs, if their representation in the local DM density is relatively large [78].

$\mathrm{S} 2$ gives rise to a more complex array of new effects. Unlike S1, it has not been studied until now; despite the fact that it also passes through the solar neighbourhood and is of roughly equal prominence as an action space substructure in Gaia-SDSS. S2 moves on a prograde, but highly polar orbit, so its DM enters the Milky Way disk from above. In the Earth frame, it contributes near the peak of the speed distribution at around $300 \mathrm{~km} \mathrm{~s}^{-1}$ (see Fig. 9). Being present at lower speeds than S1 means that it can potentially impact signals in axion haloscopes in a much more remarkable way. In fact there are important implications for the very detection of the axion. Since sharper peaks show up more strongly over thermal noise, $\mathrm{S} 2$ is positioned at the ideal frequency to enhance the sensitivity of a haloscope to the axion-photon coupling.

Streams with polar orbits imply peculiar modifications to the standard lore for time dependent signals induced by DM. Not only does S2 (in combination with several other Shards) shift the peak day of the annual modulation of the event rate of nuclear recoils, it also causes that modulation to become nonsinusoidal. Here, we have also included the often-neglected (but present) effect of gravitational focusing. Since this largely involves the modulation of the low-speed tail of the speed distribution, it is more important to take into account when considering the S2, Prograde and Low Energy Shards. We studied annual modulation in the context of $\mathrm{NaI}(\mathrm{Tl})$ experiments like DAMA/LIBRA, but these broad statements apply to the annual modulation of WIMP-like DM in general.

All of the Dark Shards have interesting and potentially detectable effects on angular and directional signals. Owing to the large quantity of retrograde material, the DM flux is enhanced in the direction of galactic rotation (towards the Cygnus constellation). This is to the benefit of direction-sensitive experiments. However, the Dark Shards on radial or polar orbits lead to additional peaks in the DM flux that have large angular separation from the expected peak towards Cygnus (compare the two panels of Fig. 7). For nuclear recoil-based experiments like CYGNUS that aim to measure this directionality, the process of elastic scattering and the finite angular resolution partially washes out this substructure. But for direction-sensitive axion searches like CASPEr and QUAX, the modifications are complex enough to warrant a dedicated study.

This paper is the first to sketch out some of the implications of the abundant substructure found by Gaia. This field of activity is likely to become more important with future data releases, as the characterisation of stellar substructures will improve over the coming years. Perhaps the most important area for future study is the relationship between stellar substructures and their associated DM components. Despite some early progress and speculation in the literature, this problem remains underexplored and is ripe for further investigation.

\section{ACKNOWLEDGMENTS}

C.A.J.O. is supported by the Grant No. FPA201565745-P from the Spanish Ministry of Economy and 
Business and the European Regional Development Fund. C.M. is supported by the Science and Technology Facilities Council (STFC) Grant No. ST/N004663/1. G.C.M. thanks the Boustany Foundation, Cambridge Commonwealth, European \& International Trust and Isaac Newton Studentship for their support of his work. The research leading to these results has received funding from the European Research Council under the European
Union's Seventh Framework Programme (FP/2007-2013) / ERC Grant Agreement No. 308024.

\section{Appendix A: Low Energy Shards}

In Table III we list the remaining Low Energy Shards that are included in our main results but not in Table II.
[1] L. Searle and R. Zinn, Astrophys. J. 225, 357 (1978).

[2] K. Freeman and J. Bland-Hawthorn, Ann. Rev. Astron. Astrophys. 40, 487 (2002), arXiv:astro-ph/0208106 [astro-ph].

[3] R. A. Ibata, G. Gilmore, and M. J. Irwin, Nature 370, 194 (1994).

[4] R. Ibata, M. Irwin, G. F. Lewis, and A. Stolte, Astrophys. J. 547, L133 (2001), arXiv:astro-ph/0004255 [astro-ph].

[5] V. Belokurov et al., Astrophys. J. 642, L137 (2006), astro-ph/0605025.

[6] V. Belokurov et al., Astrophys. J. 658, 337 (2007), arXiv:astro-ph/0605705 [astro-ph].

[7] N. Shipp et al. (DES Collaboration), Astrophys. J. 862, 114 (2018), arXiv:1801.03097 [astro-ph.GA].

[8] A. G. A. Brown et al. (Gaia), Astron. Astrophys. 595, A2 (2016), arXiv:1609.04172 [astro-ph.IM].

[9] Gaia Collaboration et al., Astron. Astrophys. 616, A1 (2018), arXiv:1804.09365.

[10] Gaia Collaboration et al., Astron. Astrophys. 595, A1 (2016), arXiv:1609.04153 [astro-ph.IM].

[11] G. C. Myeong, N. W. Evans, V. Belokurov, N. C. Amorisco, and S. Koposov, Mon. Not. R. Astron. Soc. 475, 1537 (2018), arXiv:1712.04071 [astro-ph.GA].

[12] G. C. Myeong, N. W. Evans, V. Belokurov, J. L. Sanders, and S. E. Koposov, Mon. Not. R. Astron. Soc. 478, 5449 (2018), arXiv:1804.07050 [astro-ph.GA].

[13] L. Lancaster, V. Belokurov, and N. W. Evans, Mon. Not. R. Astron. Soc. 484, 2556 (2019), arXiv:1804.09181 [astro-ph.GA].

[14] K. Malhan, R. A. Ibata, and N. F. Martin, Mon. Not. R. Astron. Soc. 481, 3442 (2018), arXiv:1804.11339 [astroph.GA].

[15] S. Meingast, J. Alves, and V. Fürnkranz, A\&A 622, L13 (2019), arXiv:1901.06387 [astro-ph.GA].

[16] H. J. Newberg and J. L. Carlin, eds., Tidal Streams in the Local Group and Beyond, Astrophysics and Space Science Library, Vol. 420 (2016).

[17] D. Carollo et al., Nature (London) 450, 1020 (2007), arXiv:0706.3005.

[18] G. C. Myeong, N. W. Evans, V. Belokurov, J. L. Sanders, and S. E. Koposov, Astrophys. J. 856, L26 (2018), arXiv:1802.03351.

[19] V. Belokurov, D. Erkal, N. W. Evans, S. E. Koposov, and A. J. Deason, Mon. Not. R. Astron. Soc. 478, 611 (2018), arXiv:1802.03414.

[20] J. T. Mackereth et al., Mon. Not. R. Astron. Soc. 482, 3426 (2019), arXiv:1808.00968 [astro-ph.GA].

[21] H. Koppelman, A. Helmi, and J. Veljanoski, Astrophys. J 860, L11 (2018), arXiv:1804.11347 [astro-ph.GA].
[22] A. Helmi, C. Babusiaux, H. H. Koppelman, D. Massari, J. Veljanoski, and A. G. A. Brown, Nature 563, 85 (2018), arXiv:1806.06038 [astro-ph.GA].

[23] J. M. D. Kruijssen, J. L. Pfeffer, M. Reina-Campos, R. A. Crain, and N. Bastian, Mon. Not. R. Astron. Soc. 486, 3180 (2019), arXiv:1806.05680 [astro-ph.GA].

[24] A. Fattahi et al., Mon. Not. R. Astron. Soc. 484, 4471 (2019), arXiv:1810.07779 [astro-ph.GA].

[25] F. Vincenzo et al., Mon. Not. R. Astron. Soc. 487, L47 (2019), arXiv:1903.03465 [astro-ph.GA].

[26] G. C. Myeong, N. W. Evans, V. Belokurov, S. E. Koposov, and J. L. Sanders, Mon. Not. R. Astron. Soc. 469, L78 (2017), arXiv:1704.01363.

[27] K. C. Schlaufman et al., Astrophys. J. 703, 2177 (2009), arXiv:0908.2627 [astro-ph.CO].

[28] K. C. Schlaufman et al., Astrophys. J. 734, 49 (2011), arXiv:1104.1424 [astro-ph.GA].

[29] K. C. Schlaufman et al., Astrophys. J. 749, 77 (2012), arXiv:1202.2360 [astro-ph.GA].

[30] M. C. Smith et al., Mon. Not. R. Astron. Soc. 399, 1223 (2009), arXiv:0904.1012 [astro-ph.GA].

[31] A. Helmi, J. Veljanoski, M. A. Breddels, H. Tian, and L. V. Sales, Astron. Astrophys. 598, A58 (2017), arXiv:1611.00222.

[32] S. R. Majewski et al., Astrophys. J. 747, L37 (2012), arXiv:1202.1832 [astro-ph.GA].

[33] J. D. Lewin and P. F. Smith, Astropart. Phys. 6, 87 (1996).

[34] S. K. Lee and A. H. G. Peter, J. Cosmol. Astropart. Phys. 1204, 029 (2012), arXiv:1202.5035 [astro-ph.CO].

[35] C. A. J. O'Hare and A. M. Green, Phys. Rev. D 90, 123511 (2014), arXiv:1410.2749 [astro-ph.CO].

[36] C. Savage, K. Freese, and P. Gondolo, Phys. Rev. D 74, 043531 (2006), astro-ph/0607121.

[37] C. A. J. O'Hare and A. M. Green, Phys. Rev. D 95, 063017 (2017), arXiv:1701.03118 [astro-ph.CO].

[38] B. J. Kavanagh and C. A. J. O'Hare, Phys. Rev. D 94, 123009 (2016), arXiv:1609.08630 [astro-ph.CO].

[39] A. H. G. Peter, Phys. Rev. D 81, 087301 (2010), arXiv:0910.4765 [astro-ph.CO].

[40] C. McCabe, Phys. Rev. D 82, 023530 (2010), arXiv:1005.0579 [hep-ph].

[41] P. J. Fox, J. Liu, and N. Weiner, Phys. Rev. D 83, 103514 (2011), arXiv:1011.1915 [hep-ph].

[42] P. J. Fox, G. D. Kribs, and T. M. P. Tait, Phys. Rev. D 83, 034007 (2011), arXiv:1011.1910 [hep-ph].

[43] A. H. G. Peter, Phys. Rev. D 83, 125029 (2011), arXiv:1103.5145 [astro-ph.CO].

[44] C. McCabe, Phys. Rev. D 84, 043525 (2011), arXiv:1107.0741 [hep-ph]. 


\begin{tabular}{|c|c|c|c|c|c|c|c|}
\hline Name & $\begin{array}{l}\text { Number } \\
\text { of stars }\end{array}$ & $\begin{array}{c}(X, Y, Z) \\
\mathrm{kpc}\end{array}$ & $\begin{array}{c}(\Delta X, \Delta Y, \Delta Z) \\
\mathrm{kpc}\end{array}$ & $\begin{array}{c}\left(v_{R}, v_{\phi}, v_{z}\right) \\
\mathrm{kms}^{-1}\end{array}$ & $\begin{array}{c}\left(\sigma_{R}, \sigma_{\phi}, \sigma_{z}\right) \\
\mathrm{km} \mathrm{s}^{-1}\end{array}$ & $\langle[\mathrm{Fe} / \mathrm{H}]\rangle$ & $\begin{array}{l}P\left(\mathbf{x}_{\odot}\right) \\
(\sigma)\end{array}$ \\
\hline NCand0 & 19 & $(9.4,-0.6,2.4)$ & $(1.6,2.1,3.0)$ & $(364.6,94.2,56.3)$ & $(27.2,15.7,41.1)$ & $-1.4 \pm 0.2$ & 0.5 \\
\hline NCand2a & 13 & $(9.6,-0.4,3.0)$ & $(1.1,2.3,3.5)$ & $(270.2,133.8,4.8)$ & $(59.5,14.3,163.4)$ & $-1.6 \pm 0.2$ & 0.9 \\
\hline $\mathrm{NCand} 2 \mathrm{~b}$ & 22 & $(10.0,0.3,2.3)$ & $(1.8,1.7,4.3)$ & $(-250.8,139.5,-28.1)$ & $(69.6,14.9,160.7)$ & $-1.6 \pm 0.2$ & 0.6 \\
\hline NCand3 & 9 & $(9.4,0.5,-0.3)$ & $(1.3,2.1,2.7)$ & $(-212.9,104.5,123.8)$ & $(30.8,8.0,192.7)$ & $-1.4 \pm 0.1$ & 0.8 \\
\hline NCand6a & 11 & $(8.1,0.2,1.1)$ & $(0.9,1.1,1.5)$ & $(-11.9,-32.9,177.6)$ & $(30.0,13.5,7.2)$ & $-2.0 \pm 0.1$ & 0.2 \\
\hline NCand6b & 10 & $(7.5,1.4,1.7)$ & $(1.0,1.6,1.4)$ & $(-3.6,-30.4,-180.5)$ & $(30.2,11.4,10.8)$ & $-2.0 \pm 0.3$ & 0.6 \\
\hline NCand7 & 11 & $(8.4,1.2,0.7)$ & $(2.7,1.7,4.6)$ & $(-178.5,-114.8,-107.3)$ & $(188.3,14.6,302.6)$ & $-1.3 \pm 0.3$ & 0.8 \\
\hline NCand8 & 7 & $(10.1,-1.3,2.6)$ & $(1.3,1.9,4.4)$ & $(26.2,375.0,-34.1)$ & $(47.9,12.8,85.4)$ & $-1.8 \pm 0.2$ & 1.0 \\
\hline NCand9a & 9 & $(6.9,0.6,2.4)$ & $(1.3,0.9,2.7)$ & $(-0.8,64.9,-189.3)$ & $(24.1,13.9,20.9)$ & $-1.8 \pm 0.4$ & 0.9 \\
\hline NCand9b & 13 & $(7.9,0.2,2.3)$ & $(1.7,1.2,3.4)$ & $(-12.2,66.8,190.6)$ & $(29.7,16.4,28.1)$ & $-2.1 \pm 0.3$ & 0.2 \\
\hline NCand10a & 23 & $(9.0,-0.4,1.5)$ & $(1.6,1.8,3.3)$ & $(-21.9,34.2,233.1)$ & $(61.7,16.8,43.9)$ & $-1.9 \pm 0.3$ & 0.1 \\
\hline NCand10b & 13 & $(9.1,0.0,0.9)$ & $(1.7,1.6,4.2)$ & $(-1.5,23.9,-186.2)$ & $(60.8,19.8,41.7)$ & $-1.8 \pm 0.4$ & 0.2 \\
\hline NCand11 & 20 & $(9.6,0.9,1.5)$ & $(2.4,2.4,4.9)$ & $(-8.5,-212.9,98.8)$ & $(72.3,21.1,89.5)$ & $-1.8 \pm 0.1$ & 0.4 \\
\hline NCand12 & 18 & $(10.3,0.7,1.7)$ & $(2.0,1.8,5.1)$ & $(1.9,128.2,-152.3)$ & $(137.9,12.7,126.9)$ & $-1.7 \pm 0.3$ & 0.4 \\
\hline NCand13a & 12 & $(7.1,0.2,1.6)$ & $(1.3,1.3,1.6)$ & $(7.9,144.6,141.2)$ & $(35.7,17.2,24.6)$ & $-1.7 \pm 0.4$ & 0.7 \\
\hline NCand13b & 17 & $(7.4,0.7,1.0)$ & $(0.7,0.7,1.7)$ & $(-0.1,163.6,-137.5)$ & $(25.4,12.7,15.4)$ & $-1.7 \pm 0.3$ & 1.1 \\
\hline NCand16 & 5 & $(11.1,-0.3,1.9)$ & $(3.0,1.3,4.0)$ & $(-75.1,-5.0,62.7)$ & $(92.3,6.6,227.0)$ & $-1.9 \pm 0.5$ & 0.5 \\
\hline NCand17 & 15 & $(10.5,0.7,2.7)$ & $(1.8,1.5,3.1)$ & $(1.5,224.8,-18.9)$ & $(86.3,29.0,95.5)$ & $-1.7 \pm 0.2$ & 1.4 \\
\hline NCand18 & 11 & $(10.3,0.9,0.3)$ & $(1.6,1.5,2.8)$ & $(71.0,-84.6,-338.4)$ & $(45.7,16.4,29.6)$ & $-2.2 \pm 0.2$ & 0.6 \\
\hline NCand19a & 13 & $(9.2,-0.2,0.9)$ & $(1.6,1.3,2.3)$ & $(-11.9,-147.8,176.3)$ & $(51.3,17.1,24.1)$ & $-2.0 \pm 0.2$ & 0.6 \\
\hline NCand19b & 10 & $(7.6,0.7,3.9)$ & $(1.0,3.0,3.7)$ & $(-1.2,-135.2,-99.6)$ & $(39.2,9.6,45.4)$ & $-1.8 \pm 0.2$ & 1.1 \\
\hline NCand20 & 9 & $(8.0,0.1,1.8)$ & $(0.9,0.8,3.3)$ & $(-34.7,-145.3,-92.9)$ & $(27.2,14.2,39.5)$ & $-2.0 \pm 0.2$ & 0.1 \\
\hline NCand21a & 15 & $(9.0,0.2,2.9)$ & $(2.2,2.2,3.5)$ & $(-6.5,-117.2,226.9)$ & $(65.2,12.6,34.0)$ & $-1.7 \pm 0.3$ & 0.3 \\
\hline NCand $21 b$ & 11 & $(9.2,0.5,2.1)$ & $(1.6,2.6,1.9)$ & $(-26.0,-113.5,-243.7)$ & $(59.0,10.6,23.3)$ & $-1.9 \pm 0.2$ & 0.4 \\
\hline NCand22 & 20 & $(9.7,-0.0,3.3)$ & $(2.4,1.8,4.0)$ & $(-19.3,-174.6,-44.4)$ & $(95.5,17.0,127.2)$ & $-1.9 \pm 0.2$ & 0.3 \\
\hline NCand23 & 8 & $(7.2,-0.1,4.8)$ & $(2.1,1.7,2.6)$ & $(-450.2,-25.4,78.9)$ & $(22.4,12.9,27.9)$ & $-1.4 \pm 0.1$ & 1.0 \\
\hline NCand24a & 7 & $(10.0,0.0,1.5)$ & $(2.3,2.0,2.5)$ & $(-80.8,253.9,-78.9)$ & $(30.5,14.9,29.9)$ & $-1.8 \pm 0.2$ & 0.5 \\
\hline NCand24b & 8 & $(10.6,0.5,2.1)$ & $(2.6,0.6,2.3)$ & $(120.3,269.2,1.6)$ & $(23.2,18.2,43.3)$ & $-1.8 \pm 0.3$ & 0.8 \\
\hline NCand25 & 8 & $(11.2,1.7,0.9)$ & $(1.7,2.8,3.4)$ & $(-95.0,199.4,-20.1)$ & $(87.1,17.8,84.7)$ & $-1.4 \pm 0.1$ & 1.8 \\
\hline NCand26 & 7 & $(10.3,1.0,4.0)$ & $(2.2,1.7,5.4)$ & $(9.2,212.6,183.7)$ & $(78.1,11.7,29.4)$ & $-1.5 \pm 0.3$ & 0.5 \\
\hline NCand27 & 17 & $(7.5,0.5,2.4)$ & $(1.4,1.6,2.3)$ & $(-18.0,-108.3,-54.5)$ & $(40.7,22.2,123.9)$ & $-2.0 \pm 0.2$ & 0.3 \\
\hline NCand28a & 12 & $(9.4,1.1,1.6)$ & $(1.8,2.2,4.0)$ & $(-299.4,28.1,-10.2)$ & $(25.9,5.2,61.5)$ & $-1.4 \pm 0.3$ & 0.8 \\
\hline NCand28b & 8 & $(9.0,-0.3,3.4)$ & $(1.0,1.5,3.1)$ & $(249.5,19.5,115.5)$ & $(67.6,7.6,118.8)$ & $-1.3 \pm 0.1$ & 1.7 \\
\hline NCand29 & 14 & $(9.2,0.9,1.2)$ & $(1.7,1.1,4.4)$ & $(-73.0,-325.6,-113.6)$ & $(166.5,27.8,92.3)$ & $-1.8 \pm 0.5$ & 0.7 \\
\hline
\end{tabular}

TABLE III. As Table II but for the Low Energy Shards category.

[45] M. T. Frandsen, F. Kahlhoefer, C. McCabe, S. Sarkar, and K. Schmidt-Hoberg, JCAP 1201, 024 (2012), arXiv:1111.0292 [hep-ph].

[46] P. Gondolo and G. B. Gelmini, JCAP 1212, 015 (2012), arXiv:1202.6359 [hep-ph].

[47] B. J. Kavanagh and A. M. Green, Phys. Rev. Lett. 111, 031302 (2013), arXiv:1303.6868 [astro-ph.CO].

[48] B. J. Kavanagh, Phys. Rev. D 89, 085026 (2014), arXiv:1312.1852 [astro-ph.CO].

[49] E. Del Nobile, G. B. Gelmini, P. Gondolo, and J.-H. Huh, JCAP 1310, 026 (2013), arXiv:1304.6183 [hep$\mathrm{ph}]$.
[50] P. J. Fox, Y. Kahn, and M. McCullough, JCAP 1410, 076 (2014), arXiv:1403.6830 [hep-ph].

[51] B. Feldstein and F. Kahlhoefer, JCAP 1408, 065 (2014), arXiv:1403.4606 [hep-ph].

[52] G. B. Gelmini, J.-H. Huh, and S. J. Witte, JCAP 1610, 029 (2016), arXiv:1607.02445 [hep-ph].

[53] P. Gondolo and S. Scopel, JCAP 1709, 032 (2017), arXiv:1703.08942 [hep-ph].

[54] G. B. Gelmini, J.-H. Huh, and S. J. Witte, JCAP 1712, 039 (2017), arXiv:1707.07019 [hep-ph].

[55] A. Ibarra and A. Rappelt, JCAP 8, 039 (2017), arXiv:1703.09168 [hep-ph]. 
[56] A. Fowlie, JCAP 1710, 002 (2017), arXiv:1708.00181 [hep-ph].

[57] A. Ibarra, B. J. Kavanagh, and A. Rappelt, JCAP 1812, 018 (2018), arXiv:1806.08714 [hep-ph].

[58] J. Herrero-García, Y. Müller, and T. Schwetz, Phys. Dark Univ. 26, 100393 (2019), arXiv:1908.07037 [hep$\mathrm{ph}$.

[59] M. G. Aartsen et al. (IceCube, PICO Collaborations), (2019), arXiv:1907.12509 [astro-ph.HE].

[60] J. Buch, J. Fan, and J. S. C. Leung, (2019), arXiv:1910.06356 [hep-ph].

[61] G. Besla, A. Peter, and N. Garavito-Camargo, (2019), arXiv:1909.04140 [astro-ph.GA].

[62] M. Lisanti, L. E. Strigari, J. G. Wacker, and R. H. Wechsler, Phys. Rev. D 83, 023519 (2011), arXiv:1010.4300 [astro-ph.CO].

[63] Y.-Y. Mao, L. E. Strigari, and R. H. Wechsler, Phys. Rev. D 89, 063513 (2014), arXiv:1304.6401 [astroph.CO].

[64] Y.-Y. Mao, L. E. Strigari, R. H. Wechsler, H.-Y. $\mathrm{Wu}$, and O. Hahn, Astrophys. J. 764, 35 (2013), arXiv:1210.2721 [astro-ph.CO].

[65] I. Butsky et al., Mon. Not. R. Astron. Soc. 462, 663 (2016), arXiv:1503.04814 [astro-ph.GA].

[66] M. Kuhlen, N. Weiner, J. Diemand, P. Madau, B. Moore, D. Potter, J. Stadel, and M. Zemp, JCAP 1002, 030 (2010), arXiv:0912.2358 [astro-ph.GA].

[67] M. Kuhlen, M. Lisanti, and D. N. Spergel, Phys. Rev. D 86, 063505 (2012), arXiv:1202.0007 [astro-ph.GA].

[68] J. Herzog-Arbeitman, M. Lisanti, P. Madau, and L. Necib, Phys. Rev. Lett. 120, 041102 (2018), arXiv:1704.04499 [astro-ph.GA].

[69] J. Herzog-Arbeitman, M. Lisanti, and L. Necib, JCAP 1804, 052 (2018), arXiv:1708.03635 [astro-ph.GA].

[70] L. Necib, M. Lisanti, and V. Belokurov, Astrophys. J. 874, 3 (2019).

[71] L. Necib, M. Lisanti, S. Garrison-Kimmel, A. Wetzel, R. Sanderson, P. F. Hopkins, C.-A. Faucher-Giguère, and D. Kereš, (2018), 10.3847/1538-4357/ab3afc, arXiv:1810.12301 [astro-ph.GA].

[72] N. W. Evans, C. A. J. O'Hare, and C. McCabe, Phys. Rev. D 99, 023012 (2019), arXiv:1810.11468 [astroph.GA].

[73] B. Ostdiek et al., (2019), arXiv:1907.06652 [astroph.GA].

[74] L. Necib et al., (2019), arXiv:1907.07190 [astro-ph.GA].

[75] L. Necib et al., (2019), arXiv:1907.07681 [astro-ph.GA].

[76] N. Bozorgnia, A. Fattahi, C. S. Frenk, A. Cheek, D. G. Cerdeno, F. A. Gómez, R. J. J. Grand, and F. Marinacci, (2019), arXiv:1910.07536 [astro-ph.GA].

[77] Z. Yuan et al., (2019), arXiv:1910.07538 [astro-ph.GA].

[78] C. A. J. O'Hare, C. McCabe, N. W. Evans, G. Myeong, and V. Belokurov, Phys. Rev. D 98, 103006 (2018), arXiv:1807.09004 [astro-ph.CO].

[79] C. A. J. O'Hare, "Darkshards," Summary available at: https://cajohare.github.io/DarkShards/. Archived at: $10.5281 /$ zenodo.3372394 (2019).

[80] C. P. Ahn et al. (SDSS Collaboration), Astrophys. J. Suppl. 203, 21 (2012), arXiv:1207.7137 [astro-ph.IM].

[81] F. Anders et al., Astron. Astrophys. 564, A115 (2014), arXiv:1311.4549 [astro-ph.GA].

[82] A. L. Luo et al., Research in Astronomy and Astrophysics 15, 1095 (2015).
[83] A. R. Casey et al., Astrophys. J. 840, 59 (2017), arXiv:1609.02914 [astro-ph.SR].

[84] A. Kunder et al., Astrophys. J. 153, 75 (2017), arXiv:1609.03210 [astro-ph.SR].

[85] Z. Ivezic et al. (SDSS Collaboration), Astrophys. J. 684, 287 (2008), arXiv:0804.3850 [astro-ph].

[86] A. J. Deason, V. Belokurov, and N. W. Evans, Mon. Not. R. Astron. Soc. 416, 2903 (2011), arXiv:1104.3220 [astro-ph.GA].

[87] M. Haywood et al., Astrophys. J. 863, 113 (2018), arXiv:1805.02617 [astro-ph.GA].

[88] L. Lancaster et al., Mon. Not. R. Astron. Soc. 486, 378 (2019), arXiv:1807.04290 [astro-ph.GA].

[89] L. L. Watkins et al., Mon. Not. R. Astron. Soc. 398, 1757 (2009), arXiv:0906.0498 [astro-ph.GA].

[90] S. A. Bird, X.-X. Xue, C. Liu, J. Shen, C. Flynn, and C. Yang, AJ 157, 104 (2019), arXiv:1805.04503 [astroph.GA].

[91] J. T. Mackereth and J. Bovy, (2019), arXiv:1910.03590 [astro-ph.GA].

[92] J. Binney, Mon. Not. R. Astron. Soc. 426, 1324 (2012), arXiv:1207.4910 [astro-ph.GA].

[93] J. L. Sanders and J. Binney, Mon. Not. R. Astron. Soc. 457, 2107 (2016), arXiv:1511.08213 [astro-ph.GA].

[94] P. J. McMillan, Mon. Not. R. Astron. Soc. 465, 76 (2017), arXiv:1608.00971.

[95] A. Stephens and A. M. Boesgaard, AJ 123, 1647 (2002).

[96] K. A. Venn et al., AJ 128, 1177 (2004), arXiv:astroph/0406120 [astro-ph].

[97] T. Matsuno, W. Aoki, and T. Suda, Astrophys. J. 874, L35 (2019), arXiv:1903.09456 [astro-ph.GA].

[98] G. C. Myeong, E. Vasiliev, G. Iorio, N. W. Evans, and V. Belokurov, Mon. Not. R. Astron. Soc. 488, 1235 (2019), arXiv:1904.03185 [astro-ph.GA].

[99] R. H. Barbá et al., Astrophys. J. 870, L24 (2019), arXiv:1812.04999 [astro-ph.GA].

[100] J. D. Simpson, Mon. Not. R. Astron. Soc. 488, 253 (2019), arXiv:1902.00447 [astro-ph.GA].

[101] K. Bekki and K. C. Freeman, Mon. Not. R. Astron. Soc. 346, L11 (2003), arXiv:astro-ph/0310348 [astro-ph].

[102] S. Knirck, A. J. Millar, C. A. J. O'Hare, J. Redondo, and F. D. Steffen, JCAP 1811, 051 (2018), arXiv:1806.05927 [astro-ph.CO].

[103] M. R. Buckley, G. Mohlabeng, and C. W. Murphy, Phys. Rev. D 100, 055039 (2019), arXiv:1905.05189 [hep-ph].

[104] J. Binney, O. Gerhard, and D. Spergel, Mon. Not. R. Astron. Soc. 288, 365 (1997), astro-ph/9609066.

[105] R. Abuter et al., (2019), arXiv:1904.05721 [astroph.GA].

[106] J. Bovy, The Astrophysical Journal Supplement Series 216, 29 (2015), arXiv:1412.3451 [astro-ph.GA].

[107] A. K. Drukier, K. Freese, and D. N. Spergel, Phys. Rev. D 33, 3495 (1986).

[108] A. J. Deason et al., Mon. Not. R. Astron. Soc. 485, 3514 (2019), arXiv:1901.02016 [astro-ph.GA].

[109] M. C. Smith, N. W. Evans, and J. H. An, Astrophys. J. 698, 1110 (2009), arXiv:0902.2709 [astro-ph.GA].

[110] A. A. Williams, V. Belokurov, A. R. Casey, and N. W. Evans, Mon. Not. R. Astron. Soc. 468, 2359 (2017), arXiv:1701.01444.

[111] N. W. Evans, R. M. Hafner, and P. T. de Zeeuw, Mon. Not. R. Astron. Soc. 286, 315 (1997), arXiv:astroph/9611162 [astro-ph]. 
[112] C. W. Purcell, A. R. Zentner, and M.-Y. Wang, JCAP 1208, 027 (2012), arXiv:1203.6617 [astro-ph.GA].

[113] C. W. Purcell et al., Nature 477, 301 (2011), arXiv:1109.2918 [astro-ph.GA].

[114] M. Niederste-Ostholt, V. Belokurov, N. W. Evans, and J. Peñarrubia, Astrophys. J. 712, 516 (2010), arXiv:1002.0266.

[115] C. Wegg, O. Gerhard, and M. Bieth, Mon. Not. R. Astron. Soc. 485, 3296 (2019), arXiv:1806.09635 [astroph.GA].

[116] A. Fattahi et al., Mon. Not. R. Astron. Soc. 484, 4471 (2019), arXiv:1810.07779 [astro-ph.GA].

[117] N. C. Amorisco, Mon. Not. R. Astron. Soc. 464, 2882 (2017), arXiv:1511.08806 [astro-ph.GA].

[118] R. Schoenrich, J. Binney, and W. Dehnen, Mon. Not. R. Astron. Soc. 403, 1829 (2010), arXiv:0912.3693 [astroph.GA].

[119] G. P. Centers et al., (2019), arXiv:1905.13650 [astroph.CO].

[120] A. Derevianko, Phys. Rev. A 97, 042506 (2018), arXiv:1605.09717 [physics.atom-ph].

[121] S. J. Asztalos et al. (ADMX Collaboration), Phys. Rev. Lett. 104, 041301 (2010), arXiv:0910.5914 [astroph.CO].

[122] N. Du et al. (ADMX Collaboration), Phys. Rev. Lett. 120, 151301 (2018), arXiv:1804.05750 [hep-ex].

[123] A. Caldwell et al. (MADMAX Working Group), Phys. Rev. Lett. 118, 091801 (2017), arXiv:1611.05865 [physics.ins-det].

[124] A. J. Millar, G. G. Raffelt, J. Redondo, and F. D. Steffen, JCAP 1701, 061 (2017), arXiv:1612.07057 [hep$\mathrm{ph}$.

[125] B. M. Brubaker et al., Phys. Rev. Lett. 118, 061302 (2017), arXiv:1610.02580 [astro-ph.CO].

[126] N. M. Rapidis, Springer Proc. Phys. 211, 45 (2018), arXiv:1708.04276 [physics.ins-det].

[127] B. M. Brubaker et al., Phys. Rev. D 96, 123008 (2017), arXiv:1706.08388 [astro-ph.IM].

[128] L. Zhong et al., Springer Proc. Phys. 211, 105 (2018), arXiv:1706.03676 [astro-ph.IM].

[129] W. Chung, Proc. Sci. CORFU2015, 047 (2016).

[130] S. Lee, J. Phys. Conf. Ser. 898, 032035 (2017).

[131] W. Chung, in Proceedings, 12th Patras Workshop on Axions, WIMPs and WISPs (PATRAS 2016): Jeju Island, South Korea, June 20-24, 2016 (2017) pp. 30-34, $[, 30(2017)]$.

[132] B. T. McAllister, G. Flower, E. N. Ivanov, M. Goryachev, J. Bourhill, and M. E. Tobar, Phys. Dark Univ. 18, 67 (2017), arXiv:1706.00209 [physics.ins-det].

[133] B. T. McAllister, G. Flower, L. E. Tobar, and M. E. Tobar, Phys. Rev. Applied 9, 014028 (2018), arXiv:1705.06028 [physics.ins-det].

[134] D. Alesini, D. Babusci, D. Di Gioacchino, C. Gatti, G. Lamanna, and C. Ligi, (2017), arXiv:1707.06010 [physics.ins-det].

[135] A. A. Melcon et al., JCAP 1805, 040 (2018), arXiv:1803.01243 [hep-ex].

[136] Y. Kahn, B. R. Safdi, and J. Thaler, Phys. Rev. Lett. 117, 141801 (2016), arXiv:1602.01086 [hep-ph].

[137] J. L. Ouellet et al., Phys. Rev. Lett. 122, 121802 (2019), arXiv:1810.12257 [hep-ex].

[138] J. L. Ouellet et al., Phys. Rev. D 99, 052012 (2019), arXiv:1901.10652 [physics.ins-det].
[139] B. T. McAllister, M. Goryachev, J. Bourhill, E. N. Ivanov, and M. E. Tobar, (2018), arXiv:1803.07755 [physics.ins-det].

[140] M. Silva-Feaver et al., Proceedings, Applied Superconductivity Conference (ASC 2016): Denver, Colorado, September 4-9, 2016, IEEE Trans. Appl. Supercond. 27, 1400204 (2016), arXiv:1610.09344 [astro-ph.IM].

[141] I. G. Irastorza and J. Redondo, Prog. Part. Nucl. Phys. 102, 89 (2018), arXiv:1801.08127 [hep-ph].

[142] J. W. Foster, N. L. Rodd, and B. R. Safdi, Phys. Rev. D 97, 123006 (2018), arXiv:1711.10489 [astro-ph.CO].

[143] M. Dine, W. Fischler, and M. Srednicki, Phys. Lett. B 104, 199 (1981).

[144] A. R. Zhitnitsky, Sov. J. Nucl. Phys. 31, 260 (1980), [Yad. Fiz.31,497(1980)].

[145] T. Braine et al. (ADMX), (2019), arXiv:1910.08638 [hep-ex].

[146] D. Budker, P. W. Graham, M. Ledbetter, S. Rajendran, and A. Sushkov, Phys. Rev. X 4, 021030 (2014), arXiv:1306.6089 [hep-ph].

[147] A. Garcon et al., (2019), 10.1126/sciadv.aax4539, arXiv:1902.04644 [hep-ex].

[148] N. Crescini et al., Eur. Phys. J. C 78, 703 (2018), [Erratum: Eur. Phys. J.C78,no.9,813(2018)], arXiv:1806.00310 [hep-ex].

[149] N. Crescini et al., Nucl. Instrum. Meth. A 842, 109 (2017), arXiv:1606.04751 [physics.ins-det].

[150] R. Barbieri et al., Phys. Dark Univ. 15, 135 (2017), arXiv:1606.02201 [hep-ph].

[151] S. Afach et al., Phys. Dark Univ. 22, 162 (2018), arXiv:1807.09391 [physics.ins-det].

[152] R. Alonso, D. Blas, and P. Wolf, JHEP 07, 069 (2019), arXiv:1810.00889 [hep-ph].

[153] P. Wolf, R. Alonso, and D. Blas, Phys. Rev. D 99, 095019 (2019), arXiv:1810.01632 [quant-ph].

[154] A. Drukier and L. Stodolsky, Phys. Rev. D 30, 2295 (1984), [,395(1984)].

[155] M. W. Goodman and E. Witten, Phys. Rev. D 31, 3059 (1985), [,325(1984)].

[156] T. Marrodan Undagoitia and L. Rauch, J. Phys. D 43, 013001 (2016), arXiv:1509.08767 [physics.ins-det].

[157] D. Tucker-Smith and N. Weiner, Phys. Rev. D 64, 043502 (2001), arXiv:hep-ph/0101138 [hep-ph].

[158] L. Baudis et al., Phys. Rev. D 88, 115014 (2013), arXiv:1309.0825 [astro-ph.CO].

[159] C. McCabe, JCAP 1605, 033 (2016), arXiv:1512.00460 [hep-ph].

[160] G. Arcadi, C. Doring, C. Hasterok, and S. Vogl, (2019), arXiv:1906.10466 [hep-ph].

[161] D. S. Akerib et al. (LUX-ZEPLIN Collaboration), (2018), arXiv:1802.06039 [astro-ph.IM].

[162] A. Tan et al. (PandaX-II Collaboration), Phys. Rev. Lett. 117, 121303 (2016), arXiv:1607.07400 [hep-ex].

[163] E. Aprile et al. (XENON Collaboration), Phys. Rev. Lett. 121, 111302 (2018), arXiv:1805.12562 [astroph.CO].

[164] K. Abe et al., Nucl. Instrum. Meth. A 716, 78 (2013), arXiv:1301.2815 [physics.ins-det].

[165] T. Suzuki et al. (XMASS Collaboration), Astropart. Phys. 110, 1 (2019), arXiv:1809.05358 [astro-ph.CO].

[166] K. Freese, J. A. Frieman, and A. Gould, Phys. Rev. D 37, 3388 (1988).

[167] K. Griest, Phys. Rev. D 37, 2703 (1988). 
[168] P. Sikivie and S. Wick, Phys. Rev. D 66, 023504 (2002), arXiv:astro-ph/0203448 [astro-ph].

[169] M. S. Alenazi and P. Gondolo, Phys. Rev. D 74, 083518 (2006), arXiv:astro-ph/0608390 [astro-ph].

[170] S. K. Lee, M. Lisanti, A. H. G. Peter, and B. R. Safdi, Phys. Rev. Lett. 112, 011301 (2014), arXiv:1308.1953 [astro-ph.CO].

[171] N. Bozorgnia and T. Schwetz, JCAP 1408, 013 (2014), arXiv:1405.2340 [astro-ph.CO].

[172] E. Del Nobile, G. B. Gelmini, and S. J. Witte, JCAP 1508, 041 (2015), arXiv:1505.07538 [hep-ph].

[173] R. Bernabei et al., Eur. Phys. J. C 73, 2648 (2013), arXiv:1308.5109 [astro-ph.GA].

[174] R. Bernabei et al., Proceedings, 7th International Conference on New Frontiers in Physics (ICNFP 2018): Kolymbari, Crete, Greece, July 4-12, 2018, Universe 4, 116 (2018), [Nucl. Phys. Atom. Energy19,no.4,307(2018)], arXiv:1805.10486 [hep-ex].

[175] E. Barbosa de Souza et al. (DM-Ice Collaboration), Phys. Rev. D 95, 032006 (2017), arXiv:1602.05939 [physics.ins-det].

[176] K. W. Kim et al. (KIMS), JHEP 03, 194 (2019), arXiv:1806.06499 [hep-ex].

[177] F. Froborg (SABRE Collaboration), Proceedings, 14th International Conference on Topics in Astroparticle and Underground Physics (TAUP 2015): Torino, Italy, September 7-11, 2015, J. Phys. Conf. Ser. 718, 042021 (2016), arXiv:1601.05307 [physics.ins-det].

[178] G. Adhikari et al., Nature 564, 83 (2018), [Erratum: Nature566,no.7742,E2(2019)].

[179] G. Adhikari et al. (COSINE-100), Phys. Rev. Lett. 123, 031302 (2019), arXiv:1903.10098 [astro-ph.IM].

[180] J. Amaré et al., Eur. Phys. J. C 79, 228 (2019), arXiv:1812.01472 [astro-ph.IM].

[181] J. Amaré et al., Phys. Rev. Lett. 123, 031301 (2019), arXiv:1903.03973 [astro-ph.IM].

[182] D. N. McKinsey, (2018), arXiv:1803.10110 [hep-ex].

[183] D. Ferenc, D. Ferenc Segedin, I. Ferenc Segedin, and M. Segedin Ferenc, (2019), arXiv:1901.02139 [physics.ins-det].

[184] R. Bernabei et al., Phys. Lett. B 389, 757 (1996).

[185] H. W. Joo, H. S. Park, J. H. Kim, S. K. Kim, Y. D. Kim, H. S. Lee, and S. H. Kim, Astropart. Phys. 108, 50 (2019), arXiv:1809.10310 [physics.ins-det].
[186] F. Mayet et al., Phys. Rept. 627, 1 (2016), arXiv:1602.03781 [astro-ph.CO].

[187] M. Leyton, S. Dye, and J. Monroe, Nature Commun. 8, 15989 (2017), arXiv:1710.06724 [physics.geo-ph].

[188] D. Mei and A. Hime, Phys. Rev. D 73, 053004 (2006), arXiv:astro-ph/0512125 [astro-ph].

[189] C. A. J. O'Hare, A. M. Green, J. Billard, E. FigueroaFeliciano, and L. E. Strigari, Phys. Rev. D 92, 063518 (2015), arXiv:1505.08061 [astro-ph.CO].

[190] C. A. J. O'Hare, B. J. Kavanagh, and A. M. Green, Phys. Rev. D 96, 083011 (2017), arXiv:1708.02959 [astro-ph.CO].

[191] A. Aleksandrov et al. (NEWS Collaboration), (2016), arXiv:1604.04199 [astro-ph.IM].

[192] N. Agafonova et al. (NEWSdm Collaboration), Eur. Phys. J. C 78, 578 (2018), arXiv:1705.00613 [astroph.CO].

[193] D. Santos et al., Proceedings, 3rd Workshop on Directional Detection of Dark Matter (CYGNUS 2011), EAS Publ. Ser. 53, 25 (2012), arXiv:1111.1566 [astro-ph.IM].

[194] Q. Riffard et al., in Proceedings, 48th Rencontres de Moriond on Very High Energy Phenomena in the Universe (2013) pp. 227-230, arXiv:1306.4173 [astroph.IM].

[195] K. Nakamura et al., PTEP 2015, $043 F 01$ (2015).

[196] J. Monroe (DMTPC Collaboration), AIP Conf. Proc. 1441, 515 (2012), arXiv:1111.0220 [physics.ins-det].

[197] M. Leyton (DMTPC Collaboration), J. Phys. Conf. Ser. 718, 042035 (2016).

[198] E. Daw et al., Proceedings, 3rd Workshop on Directional Detection of Dark Matter (CYGNUS 2011), EAS Publ. Ser. 53, 11 (2012), arXiv:1110.0222 [physics.ins-det].

[199] J. B. R. Battat et al. (DRIFT Collaboration), Phys. Dark Univ. 9-10, 1 (2015), arXiv:1410.7821 [hep-ex].

[200] J. B. R. Battat et al., Journal of Instrumentation 11, P10019 (2016), arXiv:1606.05364 [physics.ins-det].

[201] G. Mazzitelli et al. (CYGNO Collaboration), (2019), arXiv:1901.04190 [physics.ins-det].

[202] E. Baracchini et al., [In preparation] (2019).

[203] P. Gondolo, Phys. Rev. D 66, 103513 (2002), hep$\mathrm{ph} / 0209110$.

[204] S. Deans, The Radon transform and some of its applications (John Wiley and Sons, 1983).

[205] N. Bozorgnia, G. B. Gelmini, and P. Gondolo, JCAP 1206, 037 (2012), arXiv:1111.6361 [astro-ph.CO]. 